\title{
Calcium Release Mediated by Redox-Sensitive RyR2 Channels Has a Central Role in Hippocampal Structural Plasticity and Spatial Memory
}

\author{
Jamileth Y. More,, ${ }^{1, \star}$ Barbara A. Bruna,, ${ }^{1, *}$ Pedro E. Lobos,, José L. Galaz,, Paula L. Figueroa, \\ Silvia Namias, Gina L. Sánchez, Genaro C. Barrientos, José L. Valdés, ${ }^{1,3}$ Andrea C. Paula-Lima, ${ }^{1,4}$ \\ Cecilia Hidalgo, ${ }^{1-3,5}$ and Tatiana Adasme ${ }^{1,6}$
}

\begin{abstract}
Aims: Previous studies indicate that hippocampal synaptic plasticity and spatial memory processes entail calcium release from intracellular stores mediated by ryanodine receptor (RyR) channels. In particular, RyRmediated $\mathrm{Ca}^{2+}$ release is central for the dendritic spine remodeling induced by brain-derived neurotrophic factor (BDNF), a neurotrophin that stimulates complex signaling pathways leading to memory-associated protein synthesis and structural plasticity. To examine if upregulation of ryanodine receptor type-2 (RyR2) channels and the spine remodeling induced by BDNF entail reactive oxygen species (ROS) generation, and to test if RyR2 downregulation affects BDNF-induced spine remodeling and spatial memory.

Results: Downregulation of RyR2 expression (short hairpin RNA [shRNA]) in primary hippocampal neurons, or inhibition of nitric oxide synthase (NOS) or NADPH oxidase, prevented agonist-mediated RyR-mediated $\mathrm{Ca}^{2+}$ release, whereas BDNF promoted cytoplasmic ROS generation. RyR2 downregulation or inhibitors of N-methyl-D-aspartate (NMDA) receptors, or NOS or of NADPH oxidase type-2 (NOX2) prevented RyR2 upregulation and the spine remodeling induced by BDNF, as did incubation with the antioxidant agent $\mathrm{N}$-acetyl L-cysteine. In addition, intrahippocampal injection of RyR2-directed antisense oligodeoxynucleotides, which caused significant RyR2 downregulation, caused conspicuous defects in a memorized spatial memory task.

Innovation: The present novel results emphasize the key role of redox-sensitive $\mathrm{Ca}^{2+}$ release mediated by RyR2 channels for hippocampal structural plasticity and spatial memory.

Conclusion: Based on these combined results, we propose (i) that BDNF-induced RyR2-mediated $\mathrm{Ca}^{2+}$ release and ROS generation via NOS/NOX2 are strictly required for the dendritic spine remodeling and the RyR2 upregulation induced by BDNF, and (ii) that RyR2 channel expression is crucial for spatial memory processes. Antioxid. Redox Signal. 29, 1125-1146.
\end{abstract}

Keywords: BDNF, reactive oxygen species, $\mathrm{Ca}^{2+}$ signals, dendritic spines, NADPH oxidase, spatial memory training

\footnotetext{
${ }^{1}$ Biomedical Neuroscience Institute, ${ }^{2}$ Institute of Biomedical Sciences, and ${ }^{4}$ Institute for Research in Dental Sciences, Faculty of Medicine, Universidad de Chile, Santiago, Chile.

${ }_{5}^{3}$ Department of Neuroscience, Faculty of Medicine, Universidad de Chile, Santiago, Chile.

${ }^{5}$ Center for Exercise, Metabolism and Cancer Studies, Faculty of Medicine, Universidad de Chile, Santiago, Chile.

${ }^{6}$ Centro Integrativo de Biología y Química Aplicada, Universidad Bernardo O’Higgins, Santiago, Chile.

*Both these authors share the first authorship.
} 


\section{Innovation}

Here, we show that cross talk between ryanodine receptor type-2 (RyR2)-mediated $\mathrm{Ca}^{2+}$ release and reactive oxygen species generation is a key feature of the signaling pathways that underlie RyR2 upregulation and the structural remodeling of dendritic spines induced by brain-derived neurotrophic factor (BDNF) in primary hippocampal neurons. We also present results showing that RyR2 channels play a central role in spatial memory processes, since after RyR2 downregulation, rats exhibited strikingly defective performances in a previously memorized spatial memory task. Consequently, the decrease in ryanodine receptor (RyR) levels exhibited by early-stage Alzheimer's disease patients may be a relevant factor for the initial memory defects associated with this neurodegenerative condition.

\section{Introduction}

Q ynaptic connections are in continuous remodeling $\checkmark$ throughout life by way of synapse formation, stabilization, and elimination $(23,24)$. This remarkable neuronal property makes possible synaptic plasticity, a neuronal attribute characterized by changes in synaptic function and structure. Activity-induced changes in gene expression underlie long-lasting synaptic plasticity, the likely cellular basis of learning and memory $(43,72,84,85)$.

Neurotrophins exert key roles as stringent regulators of synaptic plasticity (91). Earlier studies demonstrated that neurotrophin-induced hippocampal synaptic plasticity exhibits an immediate requirement for protein synthesis (50). Brainderived neurotrophic factor (BDNF), a neurotrophin that exerts multiple actions, has a central role in the modulation of neuronal activity $(18,73,107)$. Increasing hippocampal neuronal activity stimulates the production and release of BDNF $(10,27,69)$. High-frequency electrical stimulation of hippocampal cultures promotes BDNF release (38), as does the application of theta-burst trains of action potentials to the same cells (62). BDNF promotes learning and memory tasks and stimulates memory persistence $(10,27,60,80,96)$.

The central role BDNF plays in synaptic plasticity processes has been described extensively (118). By binding to specific membrane receptors, BDNF promotes changes in the expression of genes involved in synaptic function (75) and plays an important role in neuronal survival and maintenance of several neuronal systems (14). Moreover, by promoting axonal and dendritic growth of neuronal processes, BDNF controls neuronal morphology through the formation and maturation of glutamatergic and GABAergic synapses $(19,36,44,91)$. BDNF mediates pre- and postsynaptic changes related to neuronal activity, which in turn might contribute to sustained synaptic plasticity $(22,45,64,65)$. In addition, BDNF modulates synaptic efficacy by promoting presynaptic transmitter release $(5$, $105)$ and by inducing postsynaptic changes that cause longlasting increases in synaptic plasticity $(45,67)$.

Neuronal dendritic spines are the primary postsynaptic loci of excitatory synapses and have long been associated with synaptic plasticity (37). Activity-induced release of BDNF from presynaptic neurons prompts the growth of postsynaptic dendritic spines and the induction and maintenance of long-term potentiation (LTP) in hippocampal area CA1 $(10,11,27,110)$.

BDNF enhances synaptic strength by increasing the open probability of N-methyl-D-aspartate (NMDA) receptor channels via BDNF/TrkB-mediated phosphorylation of NMDA receptor subunits $(70,108)$. This effect depends specifically on the expression of the NMDA receptor NR2B subunit (66, 68). Phosphorylation of the NMDA receptor NR2B subunit, which is particularly critical for synaptic plasticity processes $(26,83,102)$, has been linked to BDNF binding to its TrkB membrane receptor during spatial memory formation via recruitment of Fyn kinase (79). In the hippocampus, BDNF activates $\mathrm{Na}^{+}$inward currents mediated by $\mathrm{Nav1.9}$ (17, 19, 47); the ensuing depolarization promotes $\mathrm{Ca}^{2+}$ entry via voltage-gated $\mathrm{Ca}^{2+}$ channels (61).

Binding of BDNF to TrkB triggers TrkB dimerization, promoting autophosphorylation of tyrosine residues present in the receptor intracellular domains. In turn, TrkB autophosphorylation leads to the recruitment and activation of adapter proteins involved in a range of cellular signaling pathways, including mitogen-activated kinases, phosphatidylinositol 3kinase, and phospholipase $\mathrm{C} \gamma(\mathrm{PLC} \gamma)$ (16). Hydrolysis of phosphatidylinositol 4,5-bisphosphate by activated PLC $\gamma$ generates diacylglycerol, a transient activator of protein kinase $\mathrm{C}$, and inositol 1,4,5-trisphosphate $\left(\mathrm{IP}_{3}\right)$, which through activation of $\mathrm{IP}_{3}$ receptors $\left(\mathrm{IP}_{3} \mathrm{R}\right)$ triggers $\mathrm{Ca}^{2+}$ release from intracellular stores (19).

Calcium release from the endoplasmic reticulum mediated by $\mathrm{IP}_{3} \mathrm{R}$ and ryanodine receptor (RyR) channels contributes to the generation of $\mathrm{Ca}^{2+}$ signals in response to neuronal activation [reviewed in Baker et al. (9), Johenning et al. (46), and Paula-Lima et al. (93)]. Both channels types, and RyR channels in particular, mediate $\mathrm{Ca}^{2+}$-induced $\mathrm{Ca}^{2+}$ release (CICR), a cellular process with a potential role as an amplification mechanism of postsynaptic $\mathrm{Ca}^{2+}$ entry signals (13). Immunohistological techniques have revealed heterogeneous $\mathrm{IP}_{3} \mathrm{R}$ and $\mathrm{RyR}$ expression patterns within neuronal cells of rodent brain (32). Of the three mammalian RyR isoforms, ryanodine receptor type-2 (RyR2) is the most abundant isoform expressed in rat and chicken brain $(28,31)$.

Earlier work showed that BDNF release induced by highfrequency stimulation of primary hippocampal cultures requires RyR-mediated CICR (10). BDNF also mobilizes $\mathrm{Ca}^{2+}$ from intracellular stores via $\mathrm{IP}_{3} \mathrm{R}(19,40)$, which through CICR may lead to subsequent $\mathrm{Ca}^{2+}$ signal amplification by RyR channels (1, 13, 63, 93). Moreover, BDNF elicits via TrkB a nonselective cationic current, which is insensitive to tetrodotoxin and saxitoxin and requires PLC activity and $I_{3} R$ activation; this pathway engages intracellular $\mathrm{Ca}^{2+}$ stores and extracellular $\mathrm{Ca}^{2+}$ entry, suggesting the involvement of TRPC channels in the signaling pathways induced by BDNF (5).

Adding to the complexity of the signaling pathways induced by BDNF, we reported that in primary hippocampal neurons, BDNF engages RyR channels to increase RyR2 and RyR3 protein contents and to induce dendritic spine remodeling (1). These last results agree with previous reports showing that treatment of primary hippocampal neurons with the RyR agonist caffeine promotes RyR-dependent dendritic spine remodeling $(58,59)$.

Neuronal calcium signals play key roles in memory processes (90). In particular, RyR-mediated calcium release participates 
in the acquisition and/or consolidation of spatial memory processes $(9,93)$. In addition to $\mathrm{Ca}^{2+}$ signals, reactive oxygen species (ROS), including superoxide anion, hydrogen peroxide $\left(\mathrm{H}_{2} \mathrm{O}_{2}\right)$, and hydroxyl radical, together with reactive nitrogen species (RNS), have important roles in synaptic plasticity processes associated with learning and memory $(48,49,76,106)$. To our knowledge, however, there are no reports showing enhanced ROS generation as part of the complex signaling pathways induced by BDNF in hippocampal neurons.

Here, we report that BDNF promotes ROS generation; this ROS increase is required for BDNF-induced RyR2 upregulation and structural plasticity. Moreover, hippocampal RyR2 downregulation prevented BDNF-induced RyR2 upregulation and dendritic spine remodeling in primary hippocampal neurons, and significantly impaired the performance of rats in a memorized spatial memory task.

\section{Results}

Downregulation of the RyR2 isoform significantly inhibits RyR-mediated calcium release

To test the specific involvement of the RyR2 isoform on BDNF-induced synaptic plasticity, we decreased RyR2 expression by transfection of neuron-enriched primary hippocampal cultures (1) with a specific short hairpin RNA (shRNA) against RyR2 (shRyR2).

Immunofluorescence analysis of neuronal cells expressing a red fluorescent protein (RFP) coded in the same vector as the shRyR2 or the shRNA against the scrambled RyR2 sequence ([shScr]; Fig. 1A), indicated that $\sim 30 \%$ of the neuronal cells present in the cultures were transfected with shRyR 2 or shScr; in addition, RyR2 immunofluorescence in the neuronal soma was 70\% lower in shRyR2-RFP-positive neurons (shRyR2, RFP) when compared with shScr-RFP-positive cells (shScr, RFP), or with RFP-negative neurons transfected with shRyR2 (shRyR2) (Fig. 1B), determined by one-way analysis of variance (ANOVA; $F=12.5 ; p<0.0001$ ), followed by Tukey's post hoc test $(* * * p<0.001 ; n=4)$.

Neurons transfected with shScr displayed comparable RyR2 immunofluorescence levels as untransfected controls. Similar results were found at the level of the dendrites (Fig. 1C); statistical analysis was performed by one-way ANOVA $(F=5.0$; $p<0.01)$, followed by Tukey's post hoc test $(* * p<0.01 ; n=4)$.

The effects of transfection with shRyR2 or shScr on RyRmediated $\mathrm{Ca}^{2+}$ signals were evaluated in single transfected neurons (RFP positive) loaded with the fluorescent $\mathrm{Ca}^{2+}$ sensitive dye Fluo-4 AM.

As illustrated in a representative experiment, addition of the specific RyR agonist 4-chloro-m-cresol (4-CMC) at a concentration of $0.5 \mathrm{mM}$ generated large fluorescence signals in shScr-transfected neurons, whereas shRyR2-transfected neurons did not respond to the agonist (Fig. 2A).

In contrast, nontransfected neurons present in the same cultures exhibited a significant fluorescence increase of $2.2 \pm 0.1$-fold [mean \pm standard error (SE), $n=4$ ] in response to addition of $0.5 \mathrm{mM} 4-\mathrm{CMC}$. Addition of 4-CMC to neurons transfected with shScr caused a significant increase of maximal Fluo-4 fluorescence, normalized to the average fluorescence value recorded before agonist addition; in contrast, Fluo-4 fluorescence did not increase following 4-CMC addition to shRyR2-transfected neurons (Fig. 2B). The statistical analysis of these results was performed with unpaired
Student's $t$-test $(* * * p<0.001 ; n=4)$. Based on these combined results, we propose that the RyR2 isoform mediates 4-CMC-induced RyR-mediated $\mathrm{Ca}^{2+}$ release in primary hippocampal neurons.

\section{Inhibition of nitric oxide synthase or NADPH oxidase type-2 prevents agonist-induced RyR-mediated $\mathrm{Ca}^{2+}$ release}

Several reports indicate that ROS/RNS stimulate neuronal RyR activity $(34,35,41,121)$. Accordingly, we tested whether pharmacological inhibitors of the nitric oxide synthase (NOS) or NADPH oxidase type-2 (NOX2) enzymes affected agonistinduced RyR-mediated $\mathrm{Ca}^{2+}$ release. Primary hippocampal neurons in cultures treated with the NOS inhibitor L-NAME $(10 \mu M)$ did not display 4-CMC-induced $\mathrm{Ca}^{2+}$ release relative to control neurons (Fig. 2C, D). Likewise, treatment with gp91 ds-tat $(1.5 \mu M)$ to inhibit the NADPH oxidase NOX2 isoform (98) prevented agonist-induced $\mathrm{Ca}^{2+}$ release (Fig. 2E, F) compared to neurons treated with the inactive scrambled peptide [Gp91 ds-tat inactive scrambled peptide (gpscr), $1.5 \mu M$ ]. Statistical analysis of the results presented in Figure 2B, D, and $\mathrm{F}$ was performed with unpaired Student's $t$-test $\left({ }^{* * *} p<0.001\right.$; $n=4)$.

\section{BDNF-induced ROS generation engages RyR2 channels, NMDA receptors, and NOS and NOX2 activities}

To test whether BDNF promotes ROS generation, hippocampal cultures were transfected with the HyPer-Cyto probe to monitor cytoplasmic $\mathrm{H}_{2} \mathrm{O}_{2}$ production (12). As shown in the representative time-lapse experiment illustrated in Supplementary Figure S1A (Supplementary Data are available online at www.liebertpub.com/ars), addition of BDNF $(50 \mathrm{ng} / \mathrm{mL})$ increased probe fluorescence in control neurons, which attained a plateau several minutes after BDNF addition, but not in neurons preincubated with inhibitory ryanodine. Quantification of the maximal fluorescence intensity showed that BDNF significantly increased HyPer-Cyto fluorescence levels $(1.74 \pm 0.08)$ over the basal value, indicated by a segmented line in Figure 3A; of note, cultures preincubated with inhibitory ryanodine $(50 \mu M)$, which was maintained during the experiment, exhibited lower fluorescence levels $(0.41 \pm 0.1)$ than control cells (Fig. 3A).

Representative time-lapse experiments (Supplementary Fig. S1B) show that addition of BDNF $(50 \mathrm{ng} / \mathrm{mL})$ significantly increased HyPer-Cyto fluorescence in neurons transfected with shScr, whereas it caused similar reductions of fluorescence intensity in neurons transfected with shRyR2 or treated with inhibitory ryanodine. The average results illustrated in Figure 3B show that BDNF addition $(50 \mathrm{ng} / \mathrm{mL})$ significantly increased probe fluorescence $(1.59 \pm 0.02)$ in neuronal cells transfected with the shScr sequence; in contrast, neurons transfected with shRyR2 displayed a lower level of fluorescence $(0.55 \pm 0.11)$ than that exhibited by neurons transfected with shScr or by control neurons. Statistical analysis of the results presented in Figure $3 \mathrm{~A}$ and B was performed with unpaired Student's $t$-test $(* * * p<0.001 ; n=5)$.

Next, we tested whether MK801, a synaptic NMDA receptor inhibitor, affected BDNF-induced ROS generation. A representative time-lapse experiment (Supplementary Fig. S1C) shows that control cells transfected with the 
A
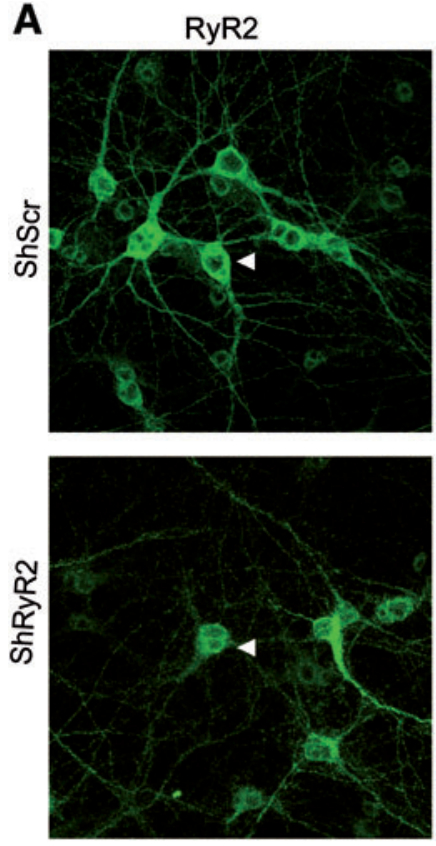

B

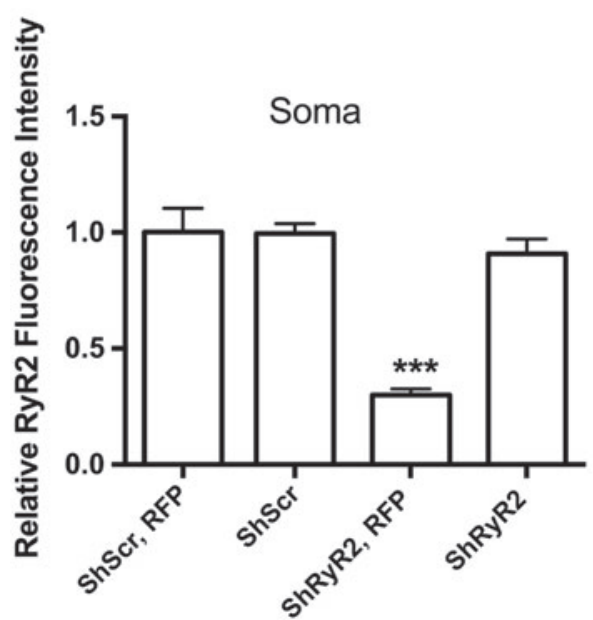

RFP
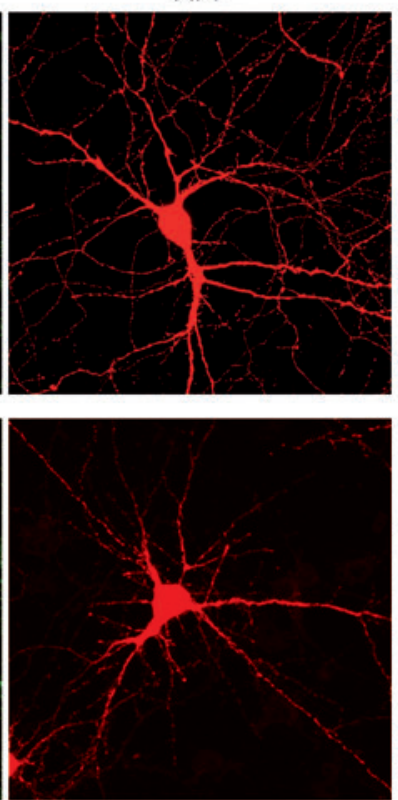

C
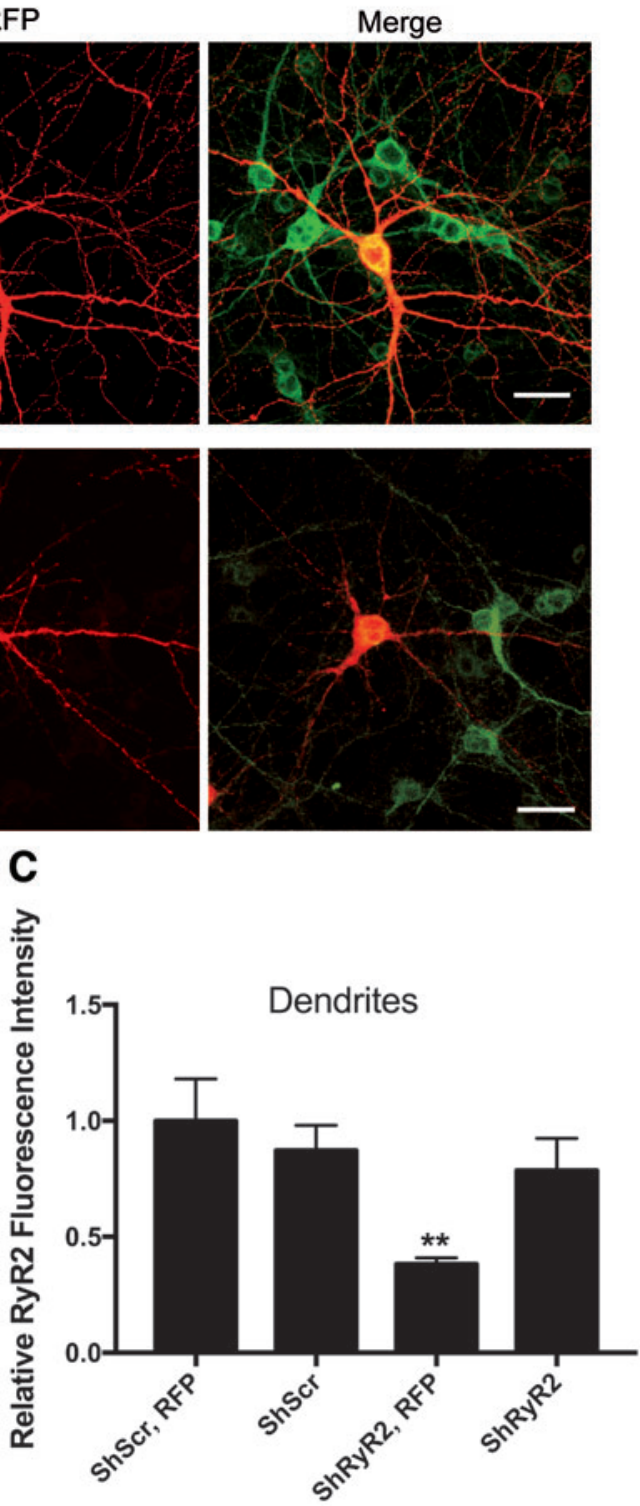

FIG. 1. Effects of shRyR2 transfection over RyR2 levels in single hippocampal neurons. (A) Representative immunofluorescence images collected $48 \mathrm{~h}$ after transfection with the shRyR2 or shScr vectors coupled to the RFP sequence. Green: RyR2 immunodetection; red: neurons expressing shRyR2 or shScr coupled to RFP. The arrowheads shown in the left panels indicate the transfected neuronal cells. Scale bar $=20 \mu \mathrm{m}$. Quantification of RyR2 green fluorescence intensity in the soma (B) or dendrites (C) of neurons expressing the shRyR2 or shScr, and in untransfected neurons. Values represent mean \pm SE $(n=4)$. Statistical analysis of the results presented in $(\mathbf{B})$ was performed with one-way ANOVA $(F=12.5$; $p<0.0001)$, followed by Tukey's post hoc test $(* * * p<0.001)$. Statistical analysis of the results presented in $(\mathbf{C})$ was performed with one-way ANOVA $(F=5.0 ; p<0.01)$, followed by Tukey's post hoc test $(* * p<0.01)$. ANOVA, analysis of variance; RFP, red fluorescent protein; RyR2, ryanodine receptor type-2; SE, standard error; shRNA, short hairpin RNA; shRyR2, shRNA against RyR2; shScr, shRNA against the scrambled RyR2 sequence. To see this illustration in color, the reader is referred to the web version of this article at www.liebertpub.com/ars

HyPer-Cyto probe displayed increased fluorescence following BDNF addition $(50 \mathrm{ng} / \mathrm{mL})$; in contrast, neuronal cells preincubated with MK801 $(10 \mu M, 30 \mathrm{~min})$ displayed a significant fluorescence decrease following BDNF addition. Quantification of results shows that while control neurons displayed a significant increase in HyPer-Cyto fluorescence following BDNF addition, neurons preincubated with MK-801 displayed a significant reduction (Fig. 3C). Statistical analysis of the results presented in Figure 3C was performed with unpaired Student's $t$-test $(* * * p<0.001 ; n=5)$.
Previous studies in primary cortical neurons have shown that stimulation of NMDA receptors promotes ROS generation via sequential stimulation of the NOS and NOX2 enzymes (31). Accordingly, we tested next whether NOS or NOX2 inhibitors affected BDNF-induced ROS generation. To this aim, we preincubated primary cultures transfected with the HyPer-Cyto probe for $30 \mathrm{~min}$ with L-NAME $(10 \mu M)$, a universal inhibitor of all NOS isoforms. A representative experiment (Supplementary Fig. S1D) shows that addition of BDNF (50 ng/mL) to cultures incubated with L- 

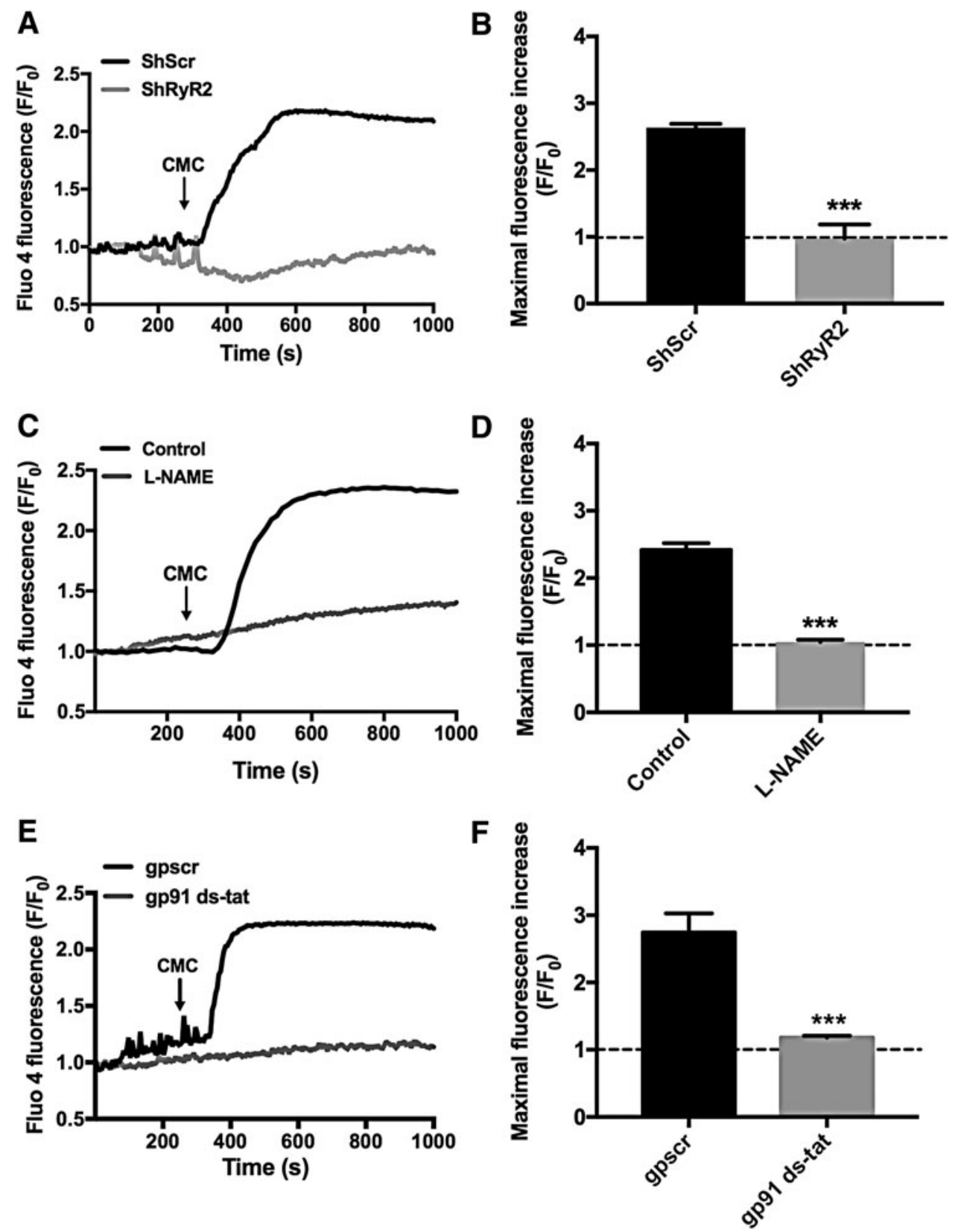

FIG. 2. RyR-mediated $\mathrm{Ca}^{2+}$ release induced by the agonist 4-CMC. Determination of fluorescence signals elicited by the RyR agonist 4-CMC in primary hippocampal cultures loaded with the $\mathrm{Ca}^{2+}$-sensing probe Fluo-4. Fluorescence signals are expressed as $F / F_{0}$, where $F_{0}$ represents the basal fluorescence recorded from several ROIs in the soma before addition of $0.5 \mathrm{~m} M$ 4-CMC. (A) Representative time-lapse experiment showing Fluo-4 fluorescence recorded in the soma of neurons transfected for $48 \mathrm{~h}$ with the shRyR2 (gray trace) or shScr (black trace) vectors coupled to the RFP sequence; addition of 4$\mathrm{CMC}$ is indicated by an arrow. Transfected neurons were identified by the red color provided by the coupled RFP vector. (B) Quantification of the results illustrated in (A). (C) Representative time-lapse experiment showing average Fluo-4 fluorescence recorded from several ROIs in the soma of neurons from cultures incubated with the NOS inhibitor L-NAME (10 $\mu \mathrm{M}$, gray trace) and from neurons present in control cultures (black trace). (D) Quantification of the results illustrated in (C). (E) Representative time-lapse experiment showing average Fluo-4 fluorescence recorded from several ROIs in the soma of neurons from cultures preincubated for $30 \mathrm{~min}$ with the NOX2 inhibitor peptide gp91 ds-tat $(1.5 \mu M$, gray trace $)$ or the control peptide gpscr $(1.5 \mu M$, black trace). (F) Quantification of the results illustrated in (E). (B, D, F) Values correspond to mean $\pm \mathrm{SE}(n=4)$. Statistical analysis of the results presented in $(\mathbf{B}, \mathbf{D}, \mathbf{F})$ was performed with unpaired Student's $t$-test $(* * * p<0.001)$. 4-CMC, 4-chloro-m-cresol; gpscr, Gp91 ds-tat inactive scrambled peptide; NOS, nitric oxide synthase; NOX2, NADPH oxidase type-2; ROI, region of optical interest; RyR, ryanodine receptor. 
NAME led to a gradual decrease of probe fluorescence with time; in comparison, control neurons displayed increased HyPer-Cyto fluorescence levels following BDNF addition. To test if NOX2 inhibition affected BDNF-induced ROS generation, we treated cultures with the NOX2 inhibitor gp91 ds-tat or with the control probe gpscr. BDNF addition $(50 \mathrm{ng} / \mathrm{mL})$ to cultures transfected with the HyPer-Cyto probe and preincubated for 30 min with gp91 ds-tat $(1.5 \mu M)$ induced a decrease in probe fluorescence with time; in contrast, BDNF addition $(50 \mathrm{ng} / \mathrm{mL})$ to cultures preincubated for $30 \mathrm{~min}$ with gpscr $(1.5 \mu M)$ caused a significant increase in probe fluorescence (Supplementary Fig. S1E). Quantification of these results shows that pharmacological inhibition of the NOS (Fig. 3D) or the NOX2 (Fig. 3E) enzymes suppressed BDNF-induced $\mathrm{H}_{2} \mathrm{O}_{2}$ generation. Statistical analysis of the results presented in Figure $3 \mathrm{D}$ and $\mathrm{E}$ was performed with unpaired Student's $t$-test $(* * * p<0.001$; $n=5)$.

A previous report in neocortical neuronal cultures described that NMDA receptor activation induces a sequential signaling cascade entailing calcium-dependent NOS activation, nitric oxide generation, increased cyclic GMP production, activation of protein kinase $\mathrm{G}(\mathrm{PKG})$, and NOX2 activation (33). A subsequent report described BDNFinduced NOS activation in primary hippocampal neurons (57). Accordingly, we tested the effects of the PKG inhibitor KT-5823 (116) on BDNF-induced ROS generation. As illustrated in Figure 3F and Supplementary Figure S1F, addition to the cultures of $5 \mu M \mathrm{KT}-582315 \mathrm{~min}$ before addition of BDNF $(50 \mathrm{ng} / \mathrm{mL})$ suppressed BDNF-induced ROS generation, showing that PKG activity mediates the ROS increase induced by BDNF. Statistical analysis of the results presented in Figure 3F was performed with unpaired Student's $t$-test $(* * * p<0.001 ; n=3)$.

Based on these combined results, we propose that BDNFinduced cytoplasmic $\mathrm{H}_{2} \mathrm{O}_{2}$ generation requires sequential NOS and NOX2 activation in response to BDNF-induced NMDA receptor stimulation, plus $\mathrm{Ca}^{2+}$ signals initially produced by $\mathrm{Ca}^{2+}$ influx via NMDA receptors and subsequently amplified through RyR2-mediated CICR.
BDNF-induced RyR2 upregulation entails RyR2-mediated $\mathrm{Ca}^{2+}$ release plus ERK1/2, NOS, and NOX activities

RyR2-mediated $\mathrm{Ca}^{2+}$ release. Previously, we reported that BDNF-induced RyR2 upregulation requires functional RyR channels (1).

To test the specific contribution of the RyR2 isoform to BDNF-induced RyR2 upregulation, primary hippocampal neurons were transfected with shRyR2 or shScr and subsequently exposed to BDNF. Transfected cultures displayed a percent reduction of RyR2 messenger RNA (mRNA) levels of $54 \pm 7(n=5)$ and a percent reduction of protein content of $38 \pm 8(n=8)$ relative to cultures transfected with shScr, as illustrated in Figure $4 \mathrm{~A}$ and $\mathrm{B}$, respectively. Following incubation with BDNF $(50 \mathrm{ng} / \mathrm{mL})$ for $6 \mathrm{~h}$, primary cultures transfected with shScr displayed a significant increase (1.8fold) in RyR2 mRNA (Fig. 4A), determined by one-way ANOVA $(F=48.0 ; p<0.0001)$, followed by Tukey's $p o s t$ hoc test $(* * * p<0.001 ; n=5)$. Likewise, RyR2 protein levels increased significantly following BDNF addition $(50 \mathrm{ng} / \mathrm{mL})$, (Fig. 4B), determined by one-way ANOVA $(F=6.6$; $p<0.005)$, followed by Tukey's post hoc test $(* p<0.05$; $\left.*^{*} p<0.01 ; n=8\right)$. Cultures transfected with shRyR2 displayed lower RyR2 mRNA and protein levels than cultures transfected with shScr; BDNF addition $(50 \mathrm{ng} / \mathrm{mL})$ increased RyR2 mRNA and protein in these cultures only to the levels exhibited by shScr-transfected cultures not treated with BDNF (Fig. 4A, B; see also original immunoblots in Supplementary Fig. S7A). These modest increments presumably reflect the response to BDNF of untransfected neurons present in the culture.

The primary hippocampal cultures used in this work displayed significant RyR2 and RyR3 mRNA levels, with average cycle threshold $(\mathrm{Ct})$ values for RyR2 and RyR3 of 24 and 26, respectively. In contrast, the average $\mathrm{Ct}$ value for RyR1 was 35, beyond the quantitative polymerase chain reaction (qPCR) detection range; this value did not reflect the use of an inadequate primer, because this primer yielded an average $\mathrm{Ct}$ value of 20 for RyR1 in rat skeletal muscle

FIG. 3. BDNF induces ROS generation in primary hippocampal neurons. Primary hippocampal cultures were transfected with the HyPer-Cyto sensor to detect changes in cytoplasmic $\mathrm{H}_{2} \mathrm{O}_{2}$ levels. Probe fluorescence was measured by confocal microscopy. Fluorescence signals are expressed as $F / F_{0}$, where $F_{0}$ represents the basal fluorescence recorded from several ROIs in the soma before addition of BDNF $(50 \mathrm{ng} / \mathrm{mL})$; values measured $2800 \mathrm{~s}$ after BDNF addition were quantified. (A) Quantification of relative HyPer-Cyto fluorescence recorded after BDNF addition (50 ng/mL) in the soma of neurons from control cultures (black bar) or from cultures preincubated for 60 min with $50 \mu M$ ryanodine to abolish RyR activity (gray bar). (B) Quantification of relative HyPer-Cyto fluorescence after BDNF addition (50 ng/mL) in the soma of neurons from cultures transfected with the vectors shScr (black bar) or shRyR2 (gray bar) coupled to the RFP sequence. Transfected neurons were identified by the red color provided by the coupled RFP vector. (C) Quantification of relative HyPer-Cyto fluorescence measured after BDNF addition $(50 \mathrm{ng} / \mathrm{mL})$ in the soma of neurons from control cultures (black bar) or from cultures preincubated for $30 \mathrm{~min}$ with the NMDA receptor inhibitor MK801 (10 $\mu M$, gray bar); this inhibitor was kept during the subsequent incubation with BDNF. (D) Quantification of relative HyPer-Cyto fluorescence measured after BDNF addition $(50 \mathrm{ng} / \mathrm{mL}$ ) in the soma of neurons from control cultures (black bar) or from cultures preincubated for 30 min with the NOS inhibitor L-NAME $(10 \mu M$, gray bar $)$; L-NAME was kept during the subsequent incubation with BDNF. (E) Quantification of relative HyPer-Cyto fluorescence measured after BDNF addition (50 ng/mL) in the soma of neurons preincubated for $30 \mathrm{~min}$ with the control gpscr peptide $(1.5 \mu M$, black bar $)$ or with the NOX2 inhibitor gp91 ds-tat peptide (1.5 $\mu \mathrm{M}$, gray bar). (F) Quantification of relative HyPer-Cyto fluorescence measured after BDNF addition (50 ng/mL) in the soma of neurons from control cultures (black bar) or from cultures preincubated for 15 min with the PKG inhibitor KT-5823 (5 $\mu M$, gray bar); this inhibitor was kept during the subsequent incubation with BDNF. Results are expressed as mean $\pm \mathrm{SE}[n=5$, except for $(\mathbf{F})$, where $n=3]$. Statistical analysis of these results was performed with unpaired Student's $t$ test $(* * * p<0.001)$. BDNF, brain-derived neurotrophic factor; $\mathrm{H}_{2} \mathrm{O}_{2}$, hydrogen peroxide; NMDA, N-methyl-D-aspartate; $\mathrm{PKG}$, protein kinase G; ROS, reactive oxygen species; Rya, ryanodine. 
A
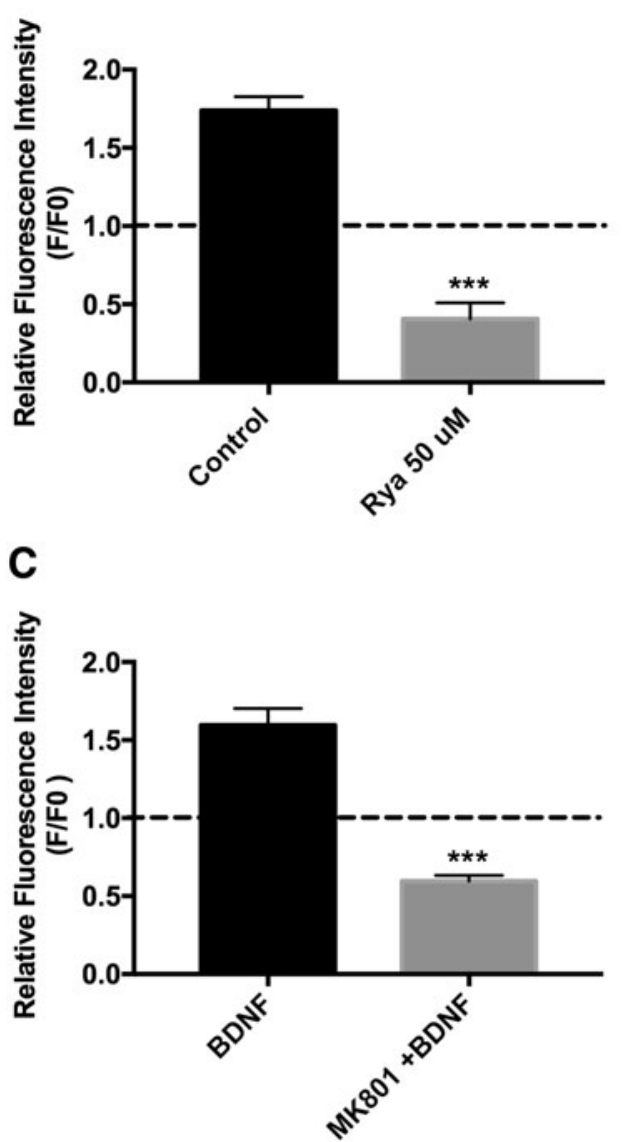

$\mathbf{E}$

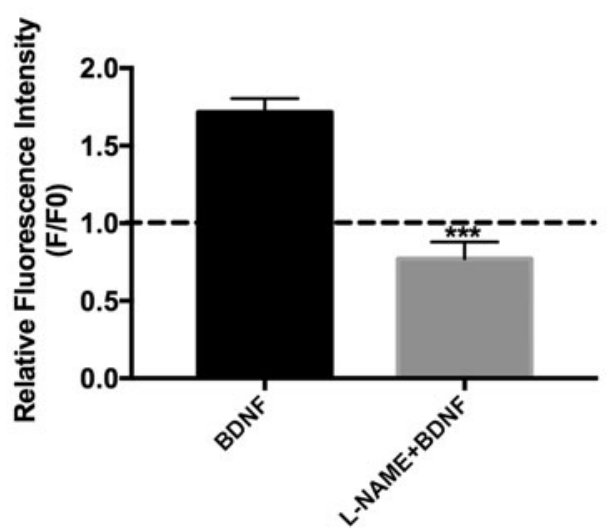

homogenates. Likewise, the primary hippocampal cultures used in this work exhibited negligible RyR1 protein levels in immunoblots performed with a highly specific RyR1 antibody (manuscript in preparation).

Cultures transfected with shRyR2 showed similar RyR3 mRNA levels as cultures transfected with shScr; incubation for $6 \mathrm{~h}$ with BDNF $(50 \mathrm{ng} / \mathrm{mL})$ produced similar increases in RyR3 mRNA levels in Src-transfected (2.2-fold) as in shRyR2transfected (2.0-fold) cultures (Supplementary Fig. S2A), showing that transfection with shRyR2 did not affect basal RyR3 mRNA levels or the increase in these levels produced by BDNF. In addition, transfection with shRyR2 did not modify basal RyR3 protein content and did not prevent the RyR3 protein increase induced by BDNF (Supplementary Fig. S2B).
B
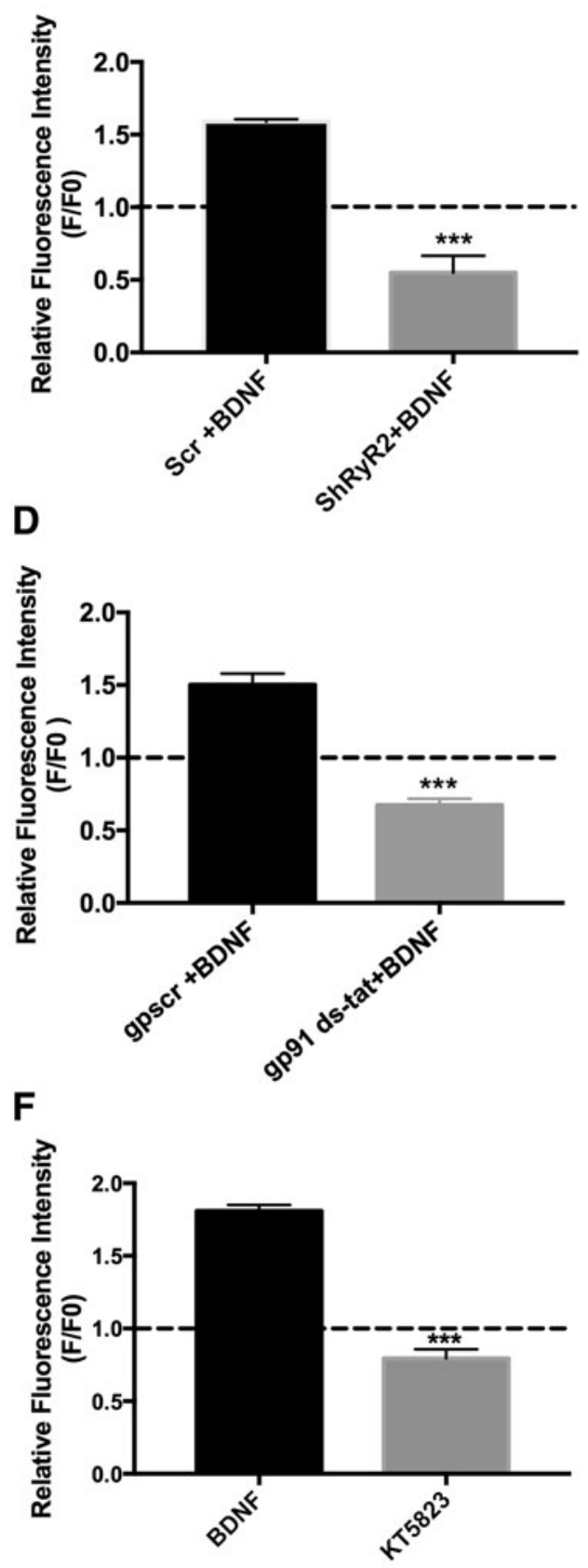

Based on these combined results, which confirm the specificity of the shRyR2 sequence used here, and on previous reports showing that the RyR2 isoform predominates in the hippocampus (31), we propose that RyR2-mediated $\mathrm{Ca}^{2+}$ release plays a prominent role in the RyR2 upregulation induced by BDNF.

ERK1/2. To prevent downstream ERK1/2 activation in response to BDNF (4), we used U0126 to inhibit the mitogenactivated protein kinase MEK. Cultures preincubated for 30 min with U0126 $(10 \mu M)$, which was maintained during the subsequent incubation with BDNF $(50 \mathrm{ng} / \mathrm{mL})$, did not exhibit increased RyR2 $\mathrm{mRNA}$ and protein levels following incubation with BDNF for $6 \mathrm{~h}$; in contrast, a significant increase in RyR2 mRNA and protein content occurred in control neurons treated 
A
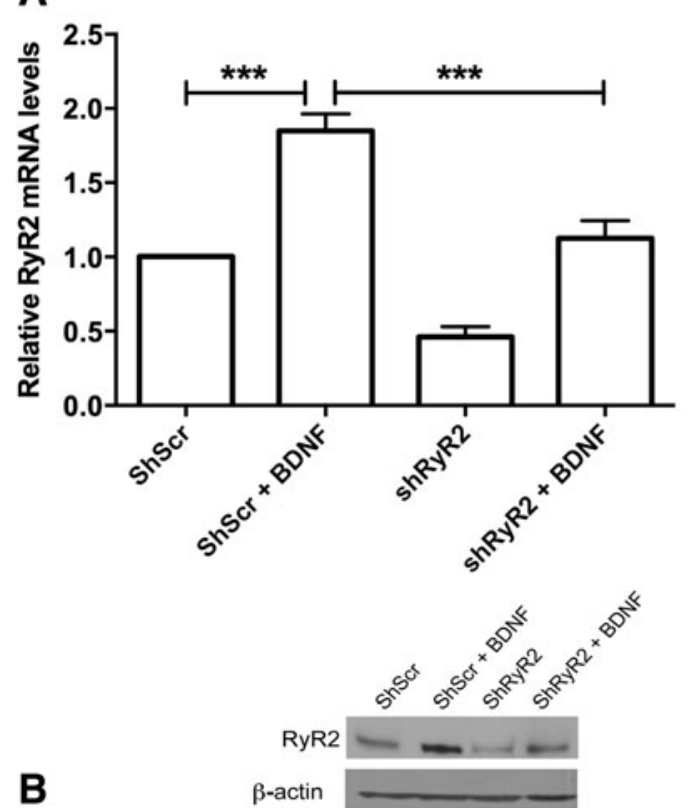

B

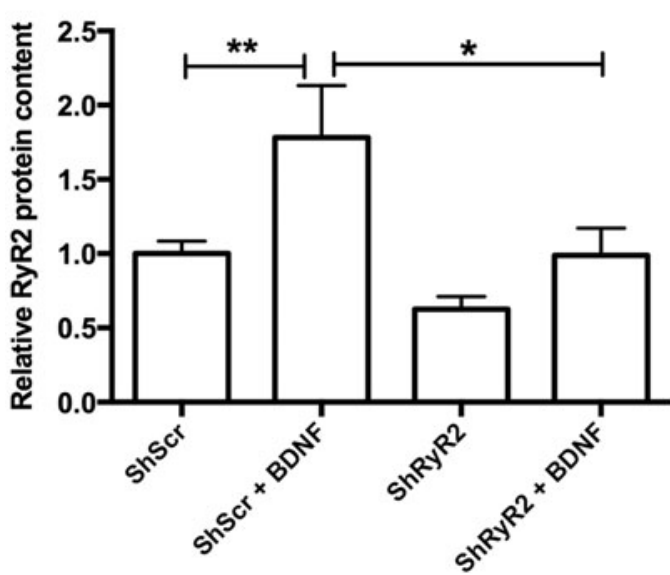

FIG. 4. Effects of BDNF and RyR2 downregulation on RyR2 mRNA and protein levels. Hippocampal neurons were transfected with shRyR2 or shScr; $48 \mathrm{~h}$ after transfection, cultures were incubated for $6 \mathrm{~h}$ with BDNF $(50 \mathrm{ng} / \mathrm{mL})$. Total mRNA and protein content was determined by qPCR and Western blot analysis, respectively; values are expressed relative to $\beta$-actin levels. (A) Quantification of RyR2 mRNA levels. (B) Quantification of RyR2 protein content. A representative blot is shown above the graph. Results are expressed as mean \pm SE. Statistical analysis of the results presented in $(\mathbf{A})$ $(n=5)$ was performed with one-way ANOVA $(F=48.0$; $p<0.0001)$, followed by Tukey's post hoc test $(* * * p<0.001)$. Statistical analysis of the results presented in (B) $(n=8)$ was performed with one-way ANOVA $(F=6.6 ; p<0.005)$, followed by Tukey's post hoc test $(* p<0.05 ; * * p<0.01)$. mRNA, messenger RNA; qPCR, quantitative polymerase chain reaction.

with BDNF (Fig. 5A, B; see also original immunoblot in Supplementary Fig. S7B). Statistical analysis of these results was performed with one-way ANOVA $(F=11.5 ; p=0.0002$ for Fig. 5A; $F=8.7$; $p=0.0003$ for Fig. 5B), followed by Tukey's post hoc test $(* * p<0.01 ; * * * p<0.001 ; n=5)$.

Reactive oxygen species. To elucidate whether BDNFinduced $\mathrm{H}_{2} \mathrm{O}_{2}$ generation contributed to BDNF-induced
RyR2 upregulation, cultures were preincubated for $30 \mathrm{~min}$ with the universal ROS scavenger $\mathrm{N}$-acetylcysteine (NAC, $10 \mathrm{~m} M$ ), which was kept during the subsequent 6-h incubation period with BDNF $(50 \mathrm{ng} / \mathrm{mL})$. Control cultures incubated with BDNF displayed increased levels of both RyR2 mRNA and protein, with values five- and twofold higher, respectively, over the controls, while NAC treatment prevented these increments (Fig. 5C, D; see also original immunoblot in Supplementary Fig. S7C). Statistical analysis of these results was performed with one-way ANOVA $(F=16.3 ; p<0.0001$ for Fig. 5C; $F=10.0 ; p=0.0007$ for Fig. 5D), followed by Tukey's post hoc test $(* p<0.05 ; * * * p<0.001 ; n=5)$.

NOS and NOX2. Cultures preincubated for $30 \mathrm{~min}$ with $\mathrm{L}_{-}$ NAME $(10 \mu M)$ and subsequently incubated with BDNF (50 ng/mL, 6h) in the presence of L-NAME did not display significant increases in RyR2 mRNA and protein levels exhibited by control cultures following BDNF addition (50 ng/ mL) (Fig. 5E, F; see also original immunoblot in Supplementary Fig. S7D). Statistical analysis of these results was performed with one-way ANOVA $(F=11.2 ; p=0.0002$ for Fig. 5E; $F=7.7 ; p=0.004$ for Fig. 5F), followed by Tukey's post hoc test $(* p<0.05 ; * * p<0.01 ; * * * p<0.001 ; n=5)$. Likewise, incubation with BDNF $(50 \mathrm{ng} / \mathrm{mL}, 6 \mathrm{~h})$ of cultures treated with the gp91 ds-tat inhibitory peptide $(1.5 \mu M$, preincubated $30 \mathrm{~min}$, kept for $6 \mathrm{~h}$ of incubation with BDNF) did not lead to RyR2 upregulation, which did occur in cultures treated with the scrambled peptide gpscr $(1.5 \mu M$, preincubated $30 \mathrm{~min}$, kept for $6 \mathrm{~h}$ of incubation with BDNF) (Fig. 5G; see also original immunoblot in Supplementary Fig. S7E). Statistical analysis of the results presented in Figure $5 \mathrm{G}$ was performed with one-way ANOVA $(F=7.4 ; p=0.0005)$, followed by Tukey's post hoc test $(* * p<0.01 ; n=5)$.

Based on these combined results, we suggest that BDNFinduced RyR2 upregulation entails a complex set of $\mathrm{Ca}^{2+}$ dependent signaling cascades, including RyR2-mediated $\mathrm{Ca}^{2+}$ release and ERK1/2, NOS, and NOX2 activation, leading to $\mathrm{H}_{2} \mathrm{O}_{2}$ generation.

\section{RyR2-mediated $\mathrm{Ca}^{2+}$ release, ROS, NOS, and NOX2 mediate BDNF-induced dendritic spine remodeling}

Changes in dendritic spine morphology induced by caffeine (58) or BDNF (1) require functional RyR channels. Here, we investigated if RyR2 downregulation affected the short-term and long-term changes in spine morphology induced by BDNF or caffeine. Addition of BDNF $(50 \mathrm{ng} / \mathrm{mL})$ to neurons transfected with shScr (detected by their green fluorescence) promoted spine elongation, as illustrated by a representative experiment (Fig. 6A). These changes, which on average took place as early as $15 \mathrm{~min}$ after BDNF addition (Fig. 6B), did not occur in neurons transfected with shRyR2, identified by their green fluorescence (Fig. 6A, B). Statistical analysis of the results presented in Figure 6B was performed with two-way ANOVA [interaction: $F_{(8,87)}=4.5$; $p=0.0001$; row factor: $F_{(4,87)}=0.7 ; p=0.57$; column factor: $\left.F_{(2,87)}=49.8 ; p=0.0001\right]$, followed by Bonferroni's post hoc test $(n=5)$. The corresponding $p$-values are given in Figure 6B legend.

To test if BDNF-induced ROS generation was required for BDNF-induced spine remodeling, we preincubated cultures for $30 \mathrm{~min}$ with $10 \mathrm{~m} M$ NAC. This treatment produced a 
A

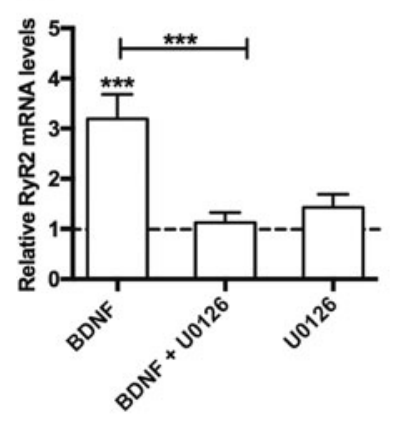

E

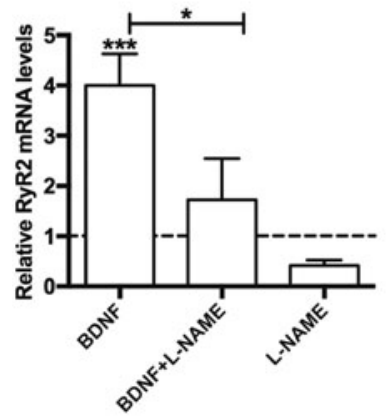

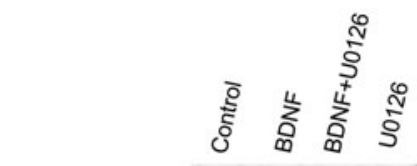
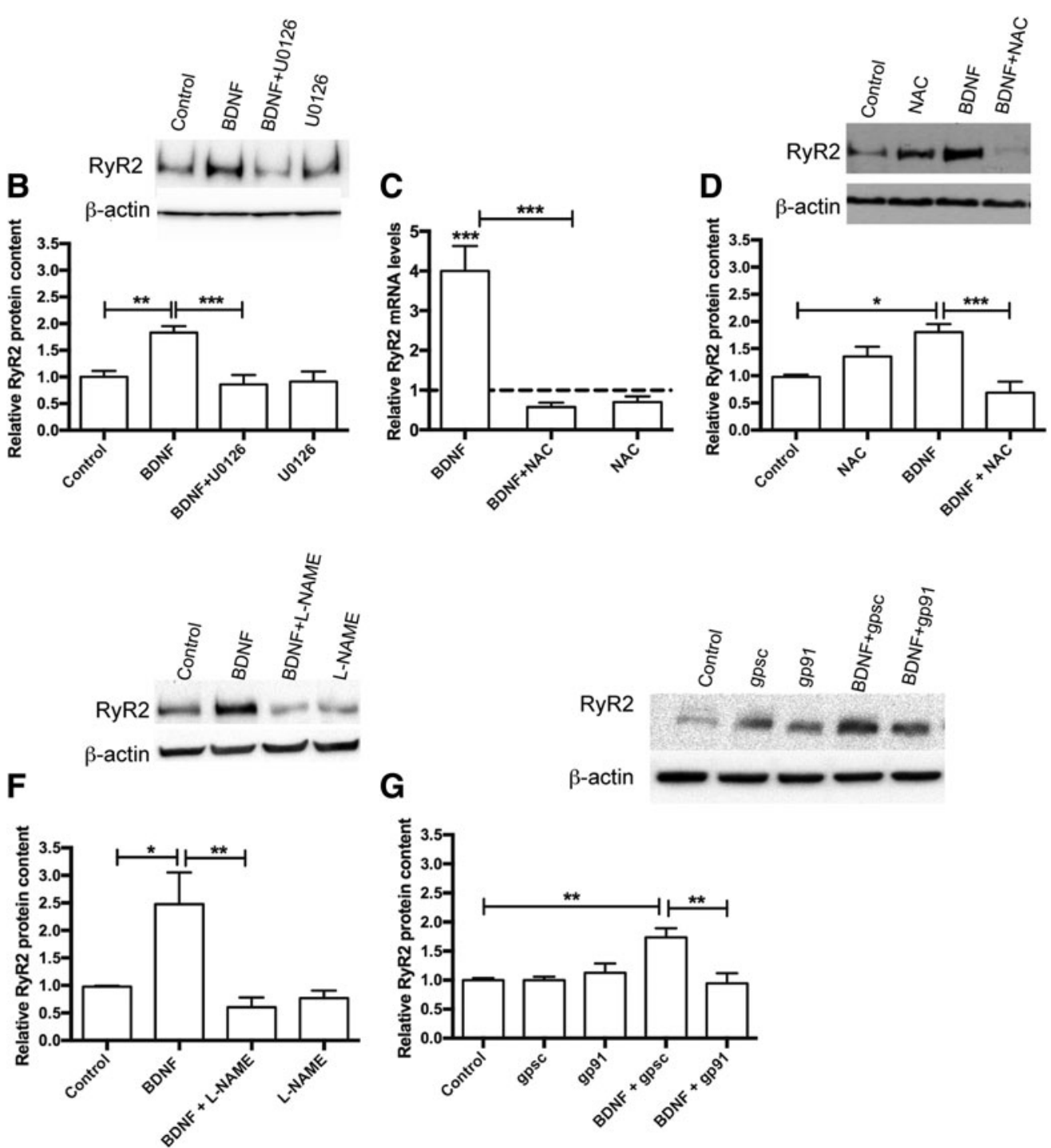
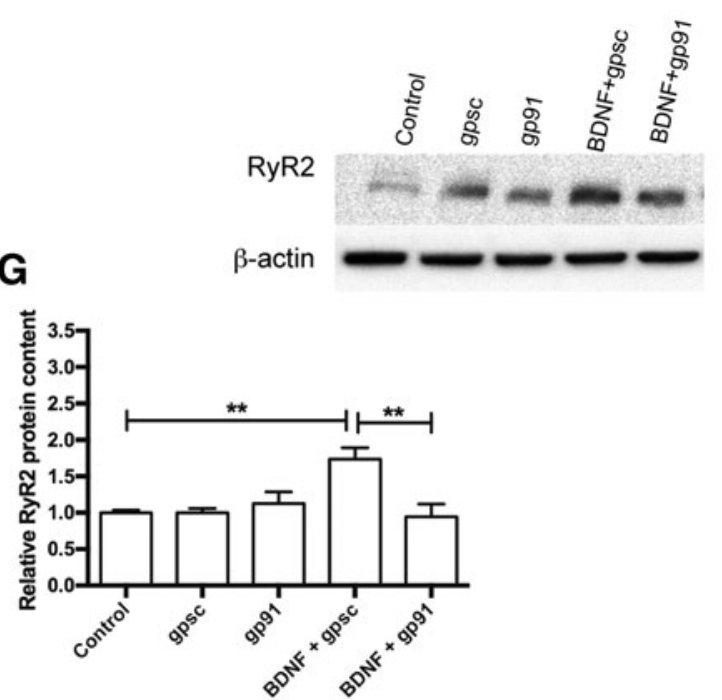

FIG. 5. BDNF-induced RyR2 upregulation requires ERK1/2, NOS, and NOX2 activities and is prevented by NAC. Primary hippocampal neurons were preincubated for $30 \mathrm{~min}$ with inhibitors of ERK1/2, NOS, or NOX2 or with NAC; inhibitors and NAC were maintained during the $6 \mathrm{~h}$ of incubation with BDNF $(50 \mathrm{ng} / \mathrm{mL})$. RyR2 mRNA levels were normalized with respect to the relative levels of $\beta$-actin. RyR2 protein content was determined by Western blot analysis; RyR2 protein band densities were quantified and normalized relative to $\beta$-actin protein levels. (A) Relative RyR2 mRNA levels and (B) relative RyR2 protein content in cultures treated with BDNF plus/minus the ERK1/2 inhibitor UO126 $(10 \mu M)$. Statistical analysis of these results was performed with one-way ANOVA $[F=11.5 ; p=0.0002$ for (A); $F=8.7$; $p=0.0003$ for (B)], followed by Tukey's post hoc test $(* * p<0.01 ; * * *<0.001)$. (C) Relative RyR2 mRNA levels and (D) relative RyR2 protein content in cultures treated with BDNF $\pm 10 \mathrm{~m} M$ NAC. Statistical analysis of these results was performed with one-way ANOVA [ $F=16.3 ; p<0.0001$ for $(\mathbf{C}) ; F=10.0 ; p=0.0007$ for (D)], followed by Tukey's post hoc test $(* p<0.05$; $* * *<0.001)$. (E) Relative RyR2 mRNA levels and (F) relative RyR2 protein content of cultures treated with BDNF plus/minus the NOS inhibitor L-NAME $(10 \mu M)$. Statistical analysis of these results was performed with oneway ANOVA $[F=11.2 ; p=0.0002$ for $(\mathbf{E}) ; F=7.7 ; p=0.004$ for $(\mathbf{F})]$, followed by Tukey's post hoc test $(* p<0.05$; $* * p<0.01$; $* * * p<0.001$ ). (G) Relative RyR2 protein content of cultures treated with BDNF plus the NOX2 inhibitor peptide gp91 ds-tat $(1.5 \mu M)$ or its inactive analog gpscr $(1.5 \mu M)$. Statistical analysis of the results presented in $(\mathbf{G})$ was performed with one-way ANOVA $(F=7.4 ; p=0.0005)$, followed by Tukey's post hoc test $(* * p<0.01)$. Results represent mean $\pm \mathrm{SE}(n=5)$. NAC, N-acetylcysteine.

significant reduction in the spine lengthening induced by BDNF (50 ng/mL) (Fig. 6C, D). Statistical analysis of the results presented in Figure 6D was performed with two-way ANOVA [interaction: $F_{(8,105)}=7.4 ; p=0.0001$; row factor: $F_{(4,105)}=18.0 ; p=0.0001$; column factor: $F_{(2,105)}=88.9$; $p=0.0001]$, followed by Dunnett's post hoc test $(n=8)$. The corresponding $p$-values are given in Figure 6D legend.
Addition of $10 \mathrm{~m} M$ caffeine to transfected cultures promoted, after $15 \mathrm{~min}$ (the first time point tested), significant spine elongation in neurons transfected with shScr but not in neurons transfected with shRyR2 (Fig. 6E, F). Statistical analysis of the results presented in Figure $6 \mathrm{~F}$ was performed with two-way ANOVA [interaction: $F_{(8,93)}=2.6 ; p<0.01$; row factor: $F_{(4,93)}=1.7 ; p=0.15$; column factor: $F_{(2,93)}=40.6$; 
$p=0.0001]$, followed by Dunnett's post hoc test $(n=8)$. The corresponding $p$-values are given in Figure $6 \mathrm{~F}$ legend. Of note, the effects of caffeine, which stimulates RyR activity directly, were faster than the BDNF-elicited changes. Presumably, BDNF stimulates RyR2-mediated $\mathrm{Ca}^{2+}$ release by engaging first other upstream signaling cascades.

In addition, NAC largely prevented the spine lengthening induced by caffeine (Fig. 6G, H). In fact, neurons treated with NAC $(10 \mathrm{~m} M)$ exhibited on average a net decrease in spine length even after addition of $10 \mathrm{~m} M$ caffeine (Fig. $6 \mathrm{H}$ ). Statistical analysis of the results presented in Figure $6 \mathrm{H}$ was performed with two-way ANOVA [interaction: $F_{(12,165)}=5.1$; $p=0.0001$; row factor: $F_{(4,165)}=2.3 ; p=0.06$; column factor: $\left.F_{(3,165)}=71.7 ; p=0.0001\right]$, followed by Dunnett's post hoc test $(n=8)$. The corresponding $p$-values are given in Figure $6 \mathrm{H}$ legend.

Next, we evaluated at long term, spine morphology changes in neurons present in cultures incubated for $6 \mathrm{~h}$ with BDNF $(50 \mathrm{ng} / \mathrm{mL})$. As illustrated in Figure $7 \mathrm{~A}$ and B, BDNF produced a marked increase in spine density in neurons transfected with shScr but not in neurons transfected with shRyR2. Statistical analysis of the results presented in Figure 7B was performed with one-way ANOVA $(F=23.0 ; p=0.0001)$, followed by Tukey's post hoc test (*** $p<0.001 ; n=5)$.

Similarly, incubation with caffeine $(10 \mathrm{~m} M, 6 \mathrm{~h})$ caused a substantial increase in spine density in neurons transfected with shScr, whereas shRyR2-transfected neurons did not undergo caffeine-induced spine remodeling (Fig. 7C, D). Statistical analysis of the results presented in Figure 7D was performed with one-way ANOVA $(F=6.8 ; p=0.002)$, followed by Tukey's post hoc test $(* p<0.05 ; * * p<0.01 ; n=5)$.

To examine whether the long-term structural plasticity changes induced by BDNF in hippocampal neurons required ROS, we preincubated the cultures for $30 \mathrm{~min}$ with NAC $(10 \mathrm{~m} M)$ before BDNF addition. NAC-treated neurons displayed a significant decrease in dendritic spine density relative to controls and did not present the density increase displayed by control neurons incubated for $6 \mathrm{~h}$ with BDNF
$(50 \mathrm{ng} / \mathrm{mL})$, (Fig. 8A, B). Statistical analysis of the results presented in Figure 8B was performed with one-way ANOVA $(F=38.1 ; p=0.0001)$, followed by Tukey's post hoc test $(* * * p<0.001 ; n=5)$.

We studied next the possible participation of NOS and NOX2 as sources of ROS generation on BDNF-induced spine remodeling. Cultures preincubated for $30 \mathrm{~min}$ with $10 \mu \mathrm{M} \mathrm{L}-$ NAME, which was maintained during the $6 \mathrm{~h}$ of incubation with BDNF $(50 \mathrm{ng} / \mathrm{mL})$, did not present an increase in spine density (Fig. 8C, D). Statistical analysis of the results presented in Figure 8D was performed with one-way ANOVA $(F=45.1 ; p=0.0001)$, followed by Tukey's post hoc test $(* * * p<0.001 ; n=5)$. Likewise, the NOX2 inhibitor gp91 dstat $(1.5 \mu M)$ prevented spine density increase produced by $6 \mathrm{~h}$ of incubation with BDNF, while neurons treated with the gpscr peptide $(1.5 \mu M)$ displayed significantly increased spine density (Fig. 8E, F). Statistical analysis of the results presented in Figure $8 \mathrm{~F}$ was performed with one-way ANOVA $(F=7.2$; $p=0.015)$, followed by Tukey's post hoc test $(* p<0.05 ; n=4)$.

We infer from these combined results that ROS generated by sequential activation of the NOS and NOX2 enzymes, together with RyR2-mediated $\mathrm{Ca}^{2+}$ release, play key roles as signaling molecules in the short- and long-term spine remodeling induced by BDNF or caffeine.

\section{Downregulation of the RyR2 isoform causes significant defects in a memorized spatial memory task}

To test if RyR2 downregulation affected spatial memory processes, we placed cannulas in the CA1 region. To evaluate hippocampal-dependent spatial learning and memory, cannulated animals (male rats) were trained during 9 consecutive sessions in the Oasis maze task, which represents a dry version of the Morris water maze $(25,53)$ and involves searching for the reward (water) in one of 21 equidistant wells on a circular arena provided with visual cues (Supplementary Fig. S3).

After the third training session, we performed bilateral injections of animals three times in a period of $25 \mathrm{~h}$ (see Table

FIG. 6. RyR2 downregulation or NAC inhibit the dendritic spine elongation induced by BDNF or caffeine. (A) Representative images of dendrites from neurons transfected with shScr (top panels) or shRyR2 (lower panels) plus GFP. (B) Quantification of spine length changes with time measured in control neurons and in neurons transfected with shRyR2 or shScr following BDNF addition. Values represent mean \pm SE $(n=5)$. Statistical analysis was performed with two-way ANOVA [interaction: $F_{(8,87)}=4.5 ; p=0.0001$; row factor: $F_{(4,87)}=0.7 ; p=0.57$; column factor: $F_{(2,87)}=49.8$; $p=0.0001]$, followed by Bonferroni's post hoc test. $* * p<0.01$ and $* * * p<0.001$ shScr plus BDNF versus shScr; ${ }^{*} \neq p<0.05$ and ${ }_{\neq \neq \neq} p<0.001$ shScr plus BDNF versus shRyR2 plus BDNF. (C) Representative images of dendrites expressing GFP preincubated for $30 \mathrm{~min}$ with $10 \mathrm{~m} M$ NAC and treated with BDNF. (D) Quantification of spine length changes with time determined in control neurons or in neurons preincubated with $10 \mathrm{~m} M$ NAC. Values represent mean \pm SE $(n=8)$. Statistical analysis was performed with two-way ANOVA [interaction: $F_{(8,105)}=7.4 ; p=0.0001$; row factor: $F_{(4,105)}=18.0 ; p=0.0001$; column factor: $F_{(2,105)}=88.9 ; p=0.0001$ ], followed by Dunnett's post hoc test. *** $p<0.001$ BDNF versus control; $\neq \neq \neq p<0.001$ BDNF versus BDNF+NAC. (E) Representative images of dendrites from neurons expressing shRyR2 or shScr+RFP incubated with $10 \mathrm{~m} M$ caffeine. (F) Quantification of spine length changes with time determined in control neurons or in neurons transfected with shRyR2 or shScr following caffeine addition. Values represent mean $\pm \mathrm{SE}(n=8)$. Statistical analysis was performed with two-way ANOVA [interaction: $F_{(8,93)}=2.6 ; p<0.01$; row factor: $F_{(4,93)}=1.7$; $p=0.15$; column factor: $\left.F_{(2,93)}=40.6 ; p=0.0001\right]$, followed by Dunnett's post hoc test $(n=5) . * * p<0.005$ and $* * * p<0.001$ shScr+caffeine versus shScr; ${ }^{\neq \neq} p<0.05$ and ${ }^{\neq \neq \neq} p<0.001$ shScr+caffeine versus shRyR $2+$ caffeine. (G) Representative images of dendrites from control neurons or from neurons preincubated for $30 \mathrm{~min}$ with $10 \mathrm{~m} M$ NAC incubated with $10 \mathrm{~m} M$ caffeine. (H) Quantification of spine length changes with time determined in control neurons and in neurons preincubated with NAC plus or minus caffeine. Values represent mean \pm SE $(n=8)$. Statistical analysis was performed with two-way ANOVA [interaction: $F_{(12,165)}=5.1 ; p=0.0001$; row factor: $F_{(4,165)}=2.3 ; p=0.06$; column factor: $F_{(3,165)}=71.7$; $p=0.0001]$, followed by Dunnett's post hoc test. $* p<0.01$ and $* * p<0.005$ caffeine versus control; ${ }^{\neq \neq \neq} p<0.001$ caffeine versus caffeine+NAC. Scale bar $=2 \mu \mathrm{m}$. GFP, green fluorescent protein. To see this illustration in color, the reader is referred to the web version of this article at www.liebertpub.com/ars 
A
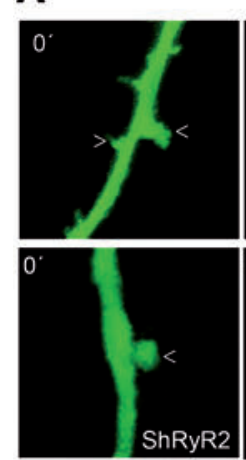

ShRyR2
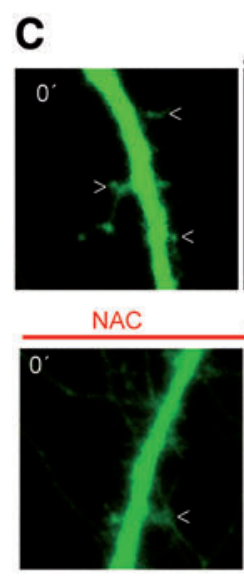

E
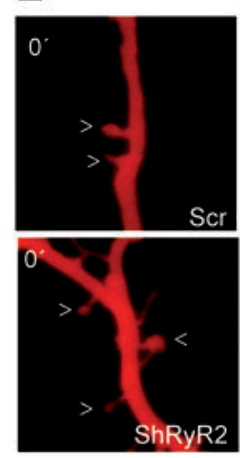

G

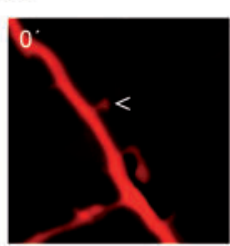

NAC

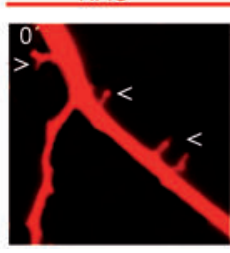

BDNF
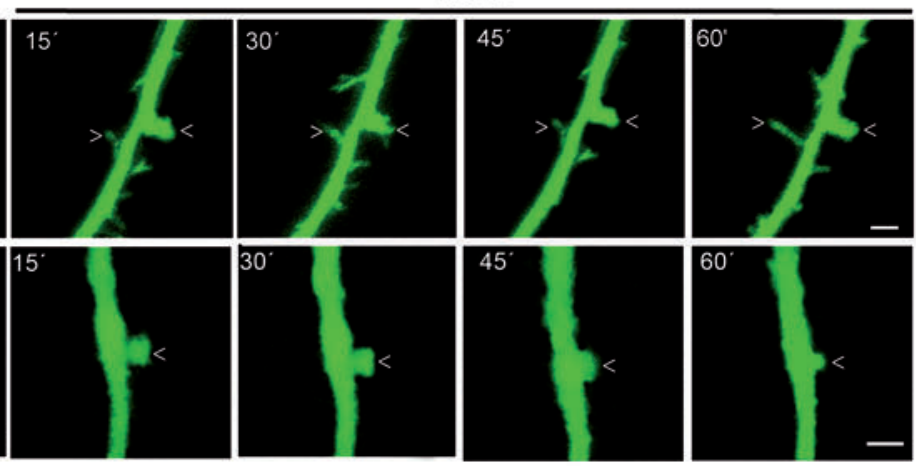

BDNF
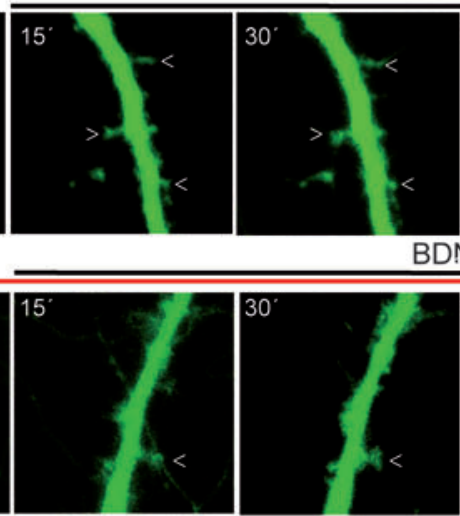

BDNF

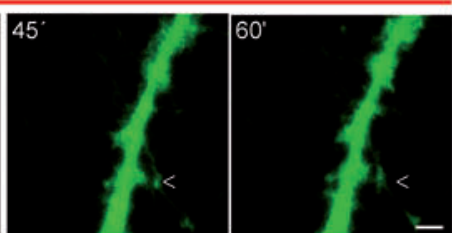

Caffeine
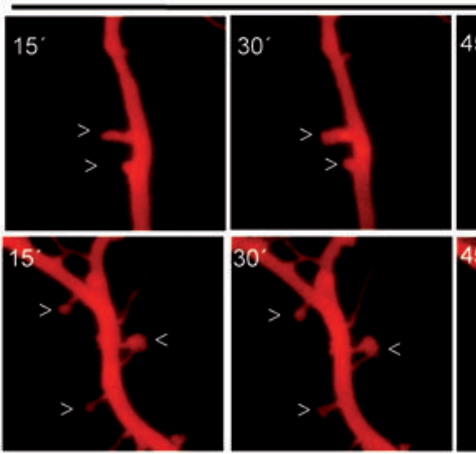

Caffeine
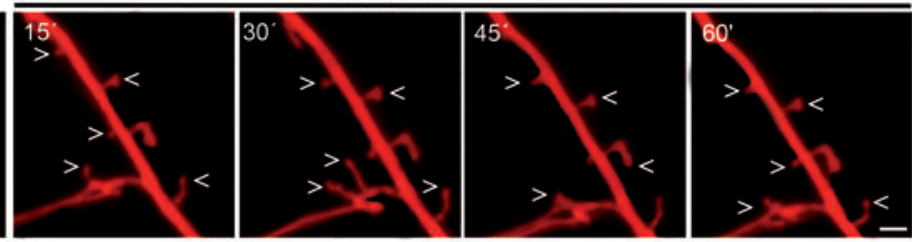

Caffeine

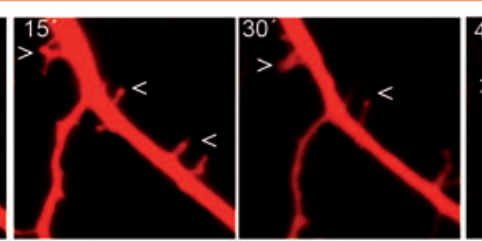

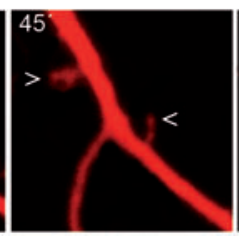

B

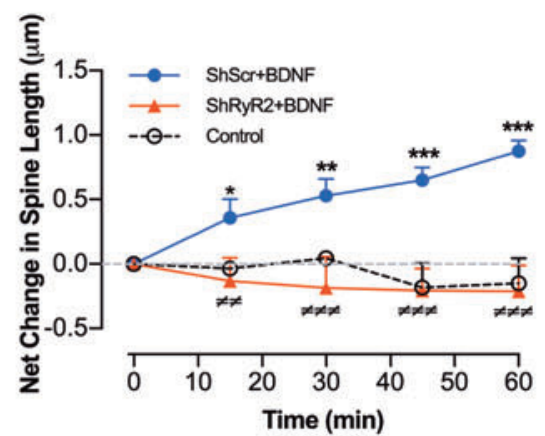

D

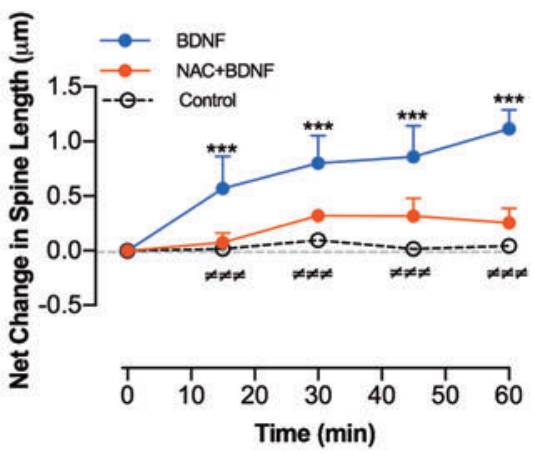

F

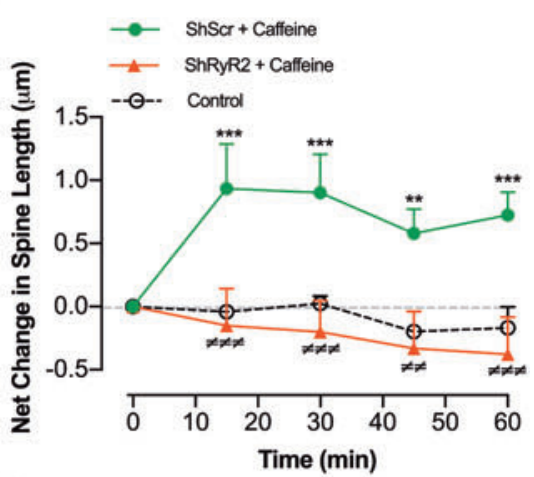

H

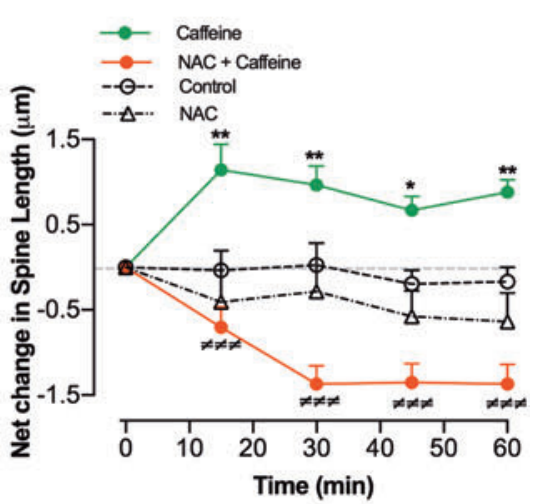



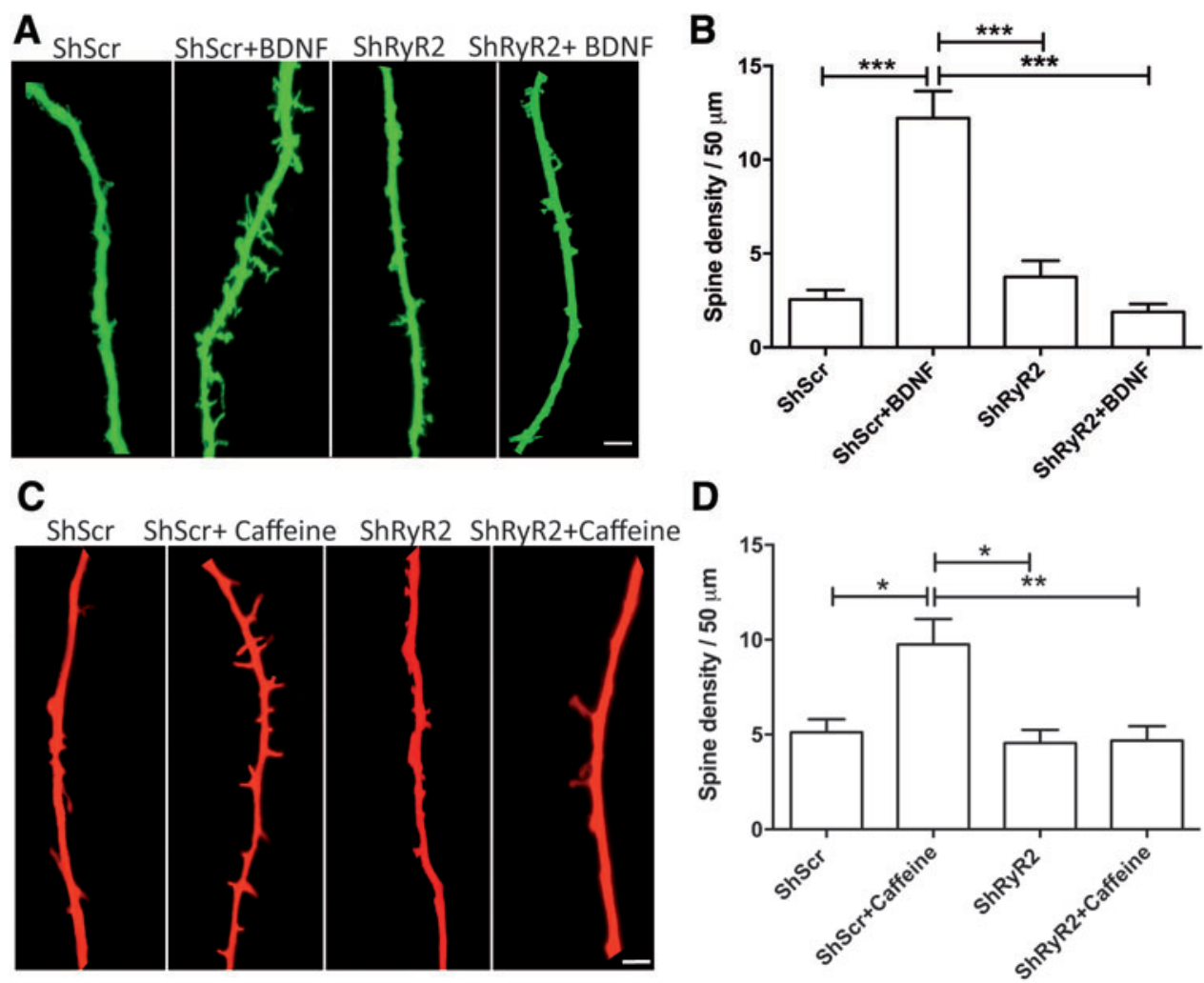

FIG. 7. RyR2 downregulation inhibits long-term dendritic spine remodeling induced by BDNF or caffeine. (A) Representative images of dendrites were acquired after incubation for $6 \mathrm{~h}$ with BDNF $(50 \mathrm{ng} / \mathrm{mL})$ or vehicle of hippocampal neurons expressing shRyR2 or shScr+GFP. (B) Quantification of spine density determined in neurons expressing shRyR2 or shScr plus or minus BDNF. Values represent mean $\pm \mathrm{SE}(n=5)$. Statistical analysis of these results was performed with one-way ANOVA $(F=23.0 ; p=0.0001)$, followed by Tukey's post hoc test $(* * * p<0.001)$. (C) Representative images of dendrites were acquired after $6 \mathrm{~h}$ of incubation with $10 \mathrm{~m} M$ caffeine or vehicle in hippocampal neurons expressing shRyR2 or shScr+RFP. (D) Spine density quantification in neurons expressing shRyR2 or shScr plus or minus caffeine. Values represent mean $\pm \operatorname{SE}(n=5)$. Statistical analysis of these results was performed with one-way ANOVA $(F=6.8 ; p=0.002)$, followed by Tukey's post hoc test $(* p<0.05 ; * * p<0.01)$. Scale bar $=2 \mu \mathrm{m}$. To see this illustration in color, the reader is referred to the web version of this article at www.liebertpub.com/ars

in Fig. 9B) with RyR2-directed antisense oligodeoxynucleotides (ODN-RyR2, $10 \mathrm{nmol} / \mu \mathrm{L}$ ) or with the scrambled sequence $(\mathrm{ODN}-\mathrm{Scr}, 10 \mathrm{nmol} / \mu \mathrm{L})$. These bilateral injections $(1 \mu \mathrm{L}$ each) caused significant downregulation of RyR2 protein contents in the CA1, CA3, and dentate gyrus hippocampal regions, determined by immunohistochemistry analysis (Supplementary Fig. S4) of tissue sections collected $6 \mathrm{~h}$ after the ninth training session, but did not affect RyR3 protein levels (Supplementary Fig. S5). Quantification of the immunofluorescence images and Western blot analysis of the whole hippocampus confirmed these findings (Supplementary Fig. S6).

We found that the animals injected with ODN-RyR2 displayed striking defects in the previously memorized spatial task, as indicated by the increased trajectories exemplified in the traces illustrated in Figure 9A, which were collected after the ninth session (see Table in Fig. 9B).

After injection, rats displayed increased ratios of observed over expected distances (Fig. 9C). Statistical analysis of the results presented in Figure 9C was performed with two-way ANOVA [interaction: $F_{(8,90)}=7.4 ; p=0.005$; row factor: $F_{(8,90)}=3.1 ; \quad p<0.0001 ;$ column factor: $F_{(1,90)}=67.4$; $p<0.0001]$. Likewise, after injection, rats displayed increased number of errors along the subsequent sessions (Fig. 9D), analyzed by two-way ANOVA [interaction:
$F_{(8,90)}=32.8 ; \quad p<0.0001 ; \quad$ row factor: $F_{(8,90)}=22.5$; $p<0.0001$; column factor: $\left.F_{(1,90)}=277.0 ; p<0.0001\right]$, followed by Bonferroni's post hoc test. Quantification of the number of errors performed in the last session by animals injected with ODN-Scr or ODN-RyR2 revealed significant differences (Fig. 9E), determined by unpaired Student's $t$ test $(* * * p<0.001 ; n=6)$.

\section{Discussion}

\section{Summary of results}

In this work, we report that RyR2 channels make a major contribution to agonist-induced RyR-mediated $\mathrm{Ca}^{2+}$ release in primary hippocampal neurons; NOS or NOX2 inhibitors abolished agonist-induced RyR-mediated $\mathrm{Ca}^{2+}$ release, confirming the redox sensitivity of this process. We also show that BDNF-induced RyR2 upregulation and dendritic spine remodeling required $\mathrm{RyR} 2$-mediated $\mathrm{Ca}^{2+}$ release, $\mathrm{H}_{2} \mathrm{O}_{2}$ generation, and NOS plus NOX2 activities. Moreover, inhibition of ERK1/2 activity prevented RyR2 upregulation induced by BDNF, whereas selective RyR2 downregulation drastically impaired the capacity of animals to perform a previously memorized spatial memory task. 

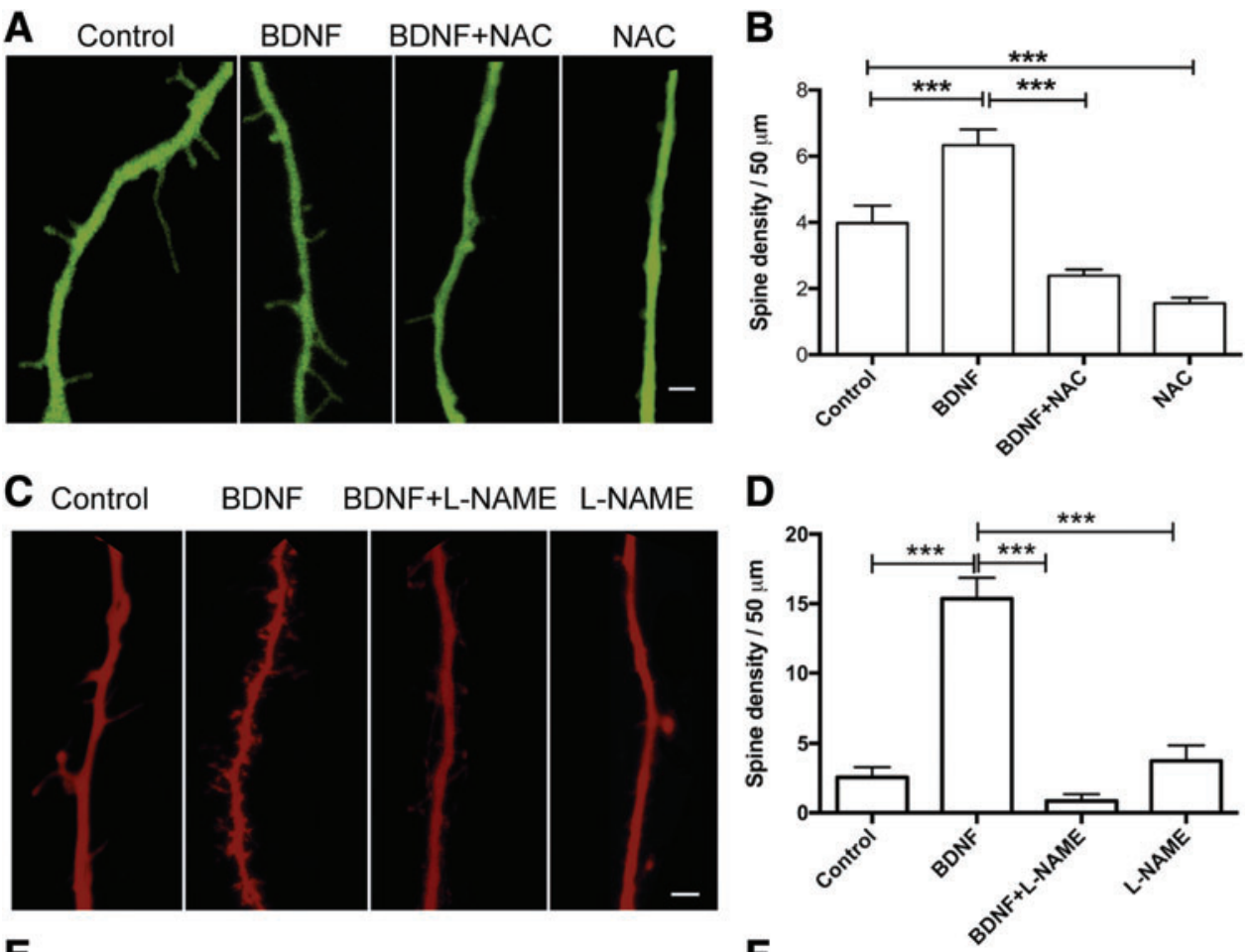

E
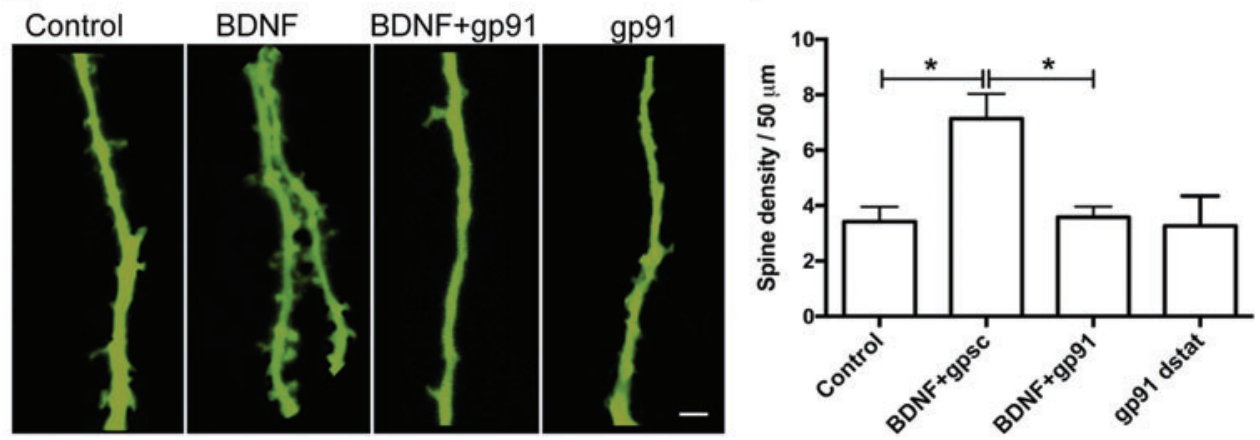

FIG. 8. Long-term structural plasticity induced by BDNF requires NOS and NOX2 ROS generation. (A) Representative images of hippocampal neurites from cultures expressing GFP incubated for $6 \mathrm{~h}$ with BDNF $(50 \mathrm{ng} / \mathrm{mL})$ or vehicle, plus or minus NAC. Neurons were preincubated for $30 \mathrm{~min}$ with $10 \mathrm{mM}$ NAC, which was maintained during the subsequent incubation for $6 \mathrm{~h}$ with BDNF or vehicle. (B) Quantification of spine density in control neurons plus or minus BDNF, and in neurons preincubated with NAC plus or minus BDNF. Values represent mean $\pm \mathrm{SE}(n=5)$. Statistical analysis of the results presented in (B) was performed with one-way ANOVA $(F=38.1 ; p=0.0001)$, followed by Tukey's post hoc test $(* * * p<0.001)$. (C) Representative images of hippocampal neurites from cultures expressing GFP and incubated for $6 \mathrm{~h}$ with vehicle plus or minus gp91 ds-tat, or with BDNF $(50 \mathrm{ng} / \mathrm{mL})$ plus gp91 ds-tat or gpscr. Cultures were preincubated with these peptides for $30 \mathrm{~min}$, and the peptides were maintained during the $6 \mathrm{~h}$ of incubation with BDNF. (D) Quantification of spine density determined in control neurons plus or minus gp91 ds-tat, and in neurons plus RFP incubated with BDNF+gp91 ds-tat or gpscr. Values represent mean \pm SE $(n=5)$. Statistical analysis of the results presented in (D) was performed with one-way ANOVA $(F=45.1 ; p=0.0001)$, followed by Tukey's post hoc test $(* * * p<0.001)$. (E) Representative images of hippocampal neurites from cultures expressing RFP and incubated for $6 \mathrm{~h}$ with vehicle plus or minus L-NAME, or with BDNF $(50 \mathrm{ng} / \mathrm{mL})$ plus or minus L-NAME. Cultures were preincubated with L-NAME $(10 \mu M)$ for $30 \mathrm{~min}$ and L-NAME was maintained during the $6 \mathrm{~h}$ of incubation with BDNF. (F) Quantification of spine density determined in control neurons plus or minus BDNF, and in neurons preincubated with L-NAME plus or minus BDNF. Values represent mean $\pm \operatorname{SE}(n=4)$. Statistical analysis of the results presented in $(\mathbf{F})$ was performed with one-way ANOVA $(F=7.2 ; p=0.015)$, followed by Tukey's post hoc test $(* p<0.05)$. Scale bar $=2 \mu \mathrm{m}$. To see this illustration in color, the reader is referred to the web version of this article at www.liebertpub.com/ars

\section{BDNF-induced ROS generation}

Previous reports showed that BDNF promotes NO generation in cortical neurons $(55,56,104)$. Rat CA1 hippocampal neurons express the NOS enzyme in dendritic spines (21), while incubation of primary hippocampal neurons with
BDNF induces NO generation in both soma and dendrites, in parallel with intracellular $\mathrm{Ca}^{2+}$ signals (57). In cortical neurons, BDNF increases the expression levels of the p47 NOX2 subunit, presumably implicating NOX2 as a downstream target of BDNF (54). In this work, we show that ROS generation forms part of the complex array of downstream 
A

ODN-Scr
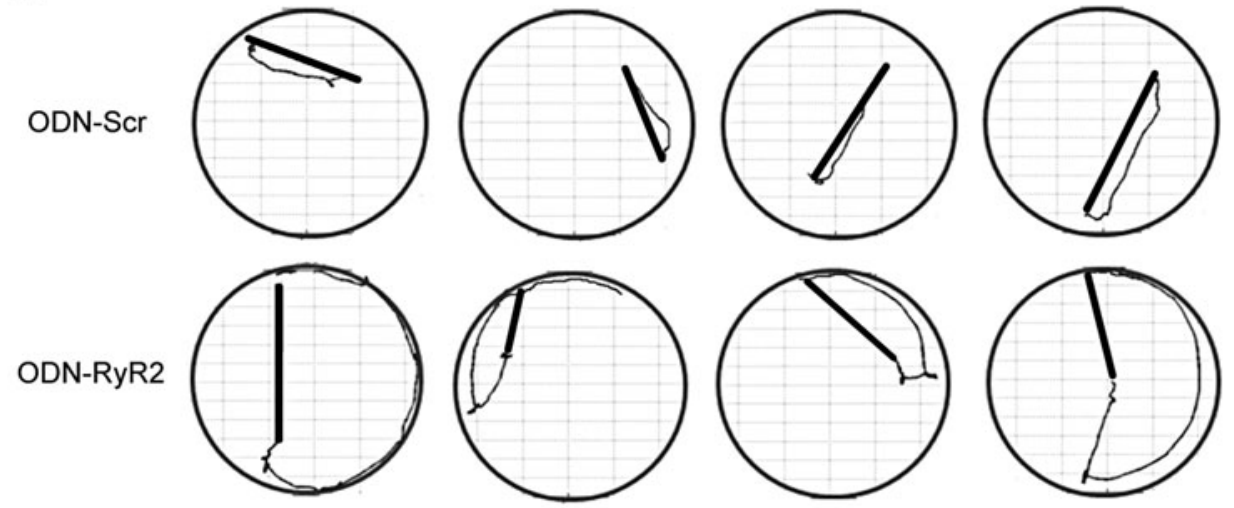

B

\begin{tabular}{|c|c|c|}
\hline & Time & Procedure \\
\hline Day 1 & $16: 00$ & Session 1 \\
\hline Day 2 & $16: 00$ & Session 2 \\
\hline Day 3 & $16: 00$ & Session 3 \\
\hline Day 4 & $10: 00$ & Injection 1 \\
\hline & $16: 00$ & Session 4 \\
\hline & $17: 00$ & Injection 2 \\
\hline Day 5 & $10: 00$ & Session 5 \\
\hline & $11: 00$ & Injection 3 \\
\hline & $16: 00$ & Session 6 \\
\hline Day 6 & $10: 00$ & Session 7 \\
\hline & $16: 00$ & Session 8 \\
\hline Day 7 & $10: 00$ & Session 9 \\
\hline & $16: 00$ & $\begin{array}{c}\text { Hippocampus } \\
\text { removed }\end{array}$ \\
\hline
\end{tabular}

C

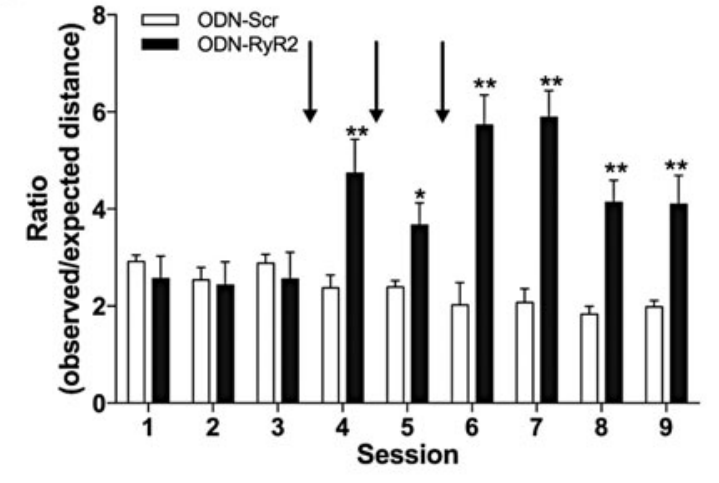

D

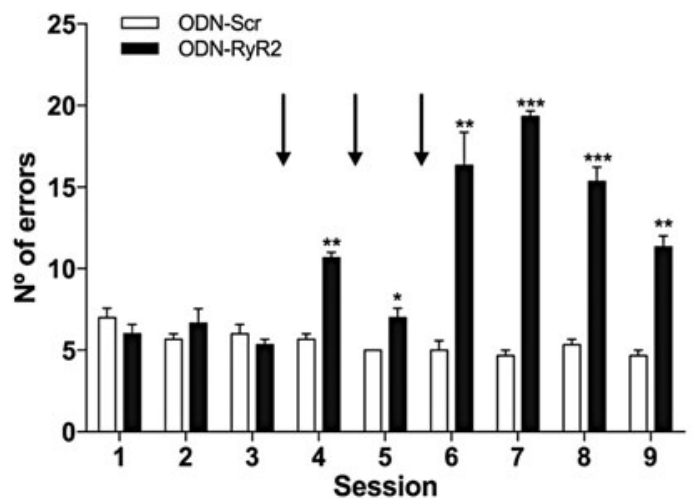

$\mathbf{E}$

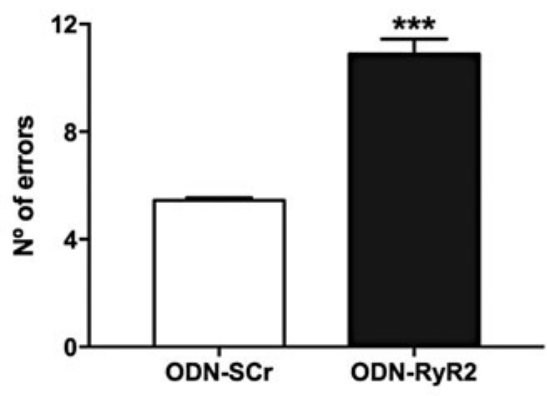

FIG. 9. RyR2 downregulation impairs spatial memory. (A) Walked (thin lines) and expected (shortest) distances (thick lines) to the reward in the arena covered by animals injected with ODN-Scr (top panels) or ODN-RyR2 (lower panels). Shown are trials 1, 5, 10, and 15 collected from session 9, as defined in (B), which shows the schedule of sessions and injections. (C) Quantification of the ratios between observed/expected distances performed by animals injected bilaterally with $1 \mu \mathrm{L}$ per injection of ODN-Scr $(10 \mathrm{nmol} / \mu \mathrm{L})$ or ODN-RyR2 $(10 \mathrm{nmol} / \mu \mathrm{L})$. The injections are indicated by the arrows. Values represent mean $\pm \operatorname{SE}(n=5)$. Statistical analysis of the results presented in $(\mathbf{C})$ was performed with two-way ANOVA [interaction: $F_{(8,90)}=7.4 ; p=0.005$; row factor: $F_{(8,90)}=3.1 ; p<0.0001$; column factor: $F_{(1,90)}=67.4 ; p<0.0001$ ] Bonferroni post hoc test $(* p<0.05 ; * *<0.01)$. (D) Quantification of the number of errors performed by animal injected as above with ODN-Scr or ODN-RyR2; the number of errors represent the times rats visited a well without the reward in each trial. The injections are indicated by arrows. Values represent mean \pm SE $(n=5)$. Two-way ANOVA [interaction: $F_{(8,90)}=32.8$; $p<0.0001$; row factor: $F_{(8.90)}=22.5 ; p<0.0001$; column factor: $F_{(1,90)}=277.0 ; p<0.0001$ ], followed by Bonferroni post hoc test $(* * p<0.01 ; * * * p<0.001)$. (E) Quantification of the number of errors performed in the last session by animals injected with ODN-Scr or ODN-RyR2. Values represent mean \pm SE $(n=6)$. Statistical analysis in $(\mathbf{E})$ was determined by unpaired Student's $t$-test $(* * * p<0.001)$. ODN-RyR2, RyR2-directed antisense oligodeoxynucleotides; ODN-Scr, scrambled sequence of RyR2-directed antisense oligodeoxynucleotides. 
signaling pathways induced by BDNF in hippocampal neurons. In physiological conditions, ROS act as signaling molecules critical for the induction of synaptic plasticity and memory formation (76). Accumulating evidence suggests that there is substantial cross talk between ROS and $\mathrm{Ca}^{2+}$ signaling in different cellular systems $(34,117)$, including hippocampal neurons (41). Here, we found that inhibition of NMDA receptors or RyR2 downregulation prevented BDNFinduced cytoplasmic $\mathrm{H}_{2} \mathrm{O}_{2}$ generation. Accordingly, we propose that $\mathrm{Ca}^{2+}$ signals initially produced by $\mathrm{Ca}^{2+}$ influx via NMDA receptors, and subsequently amplified through RyR2mediated CICR, are essential for BDNF-induced ROS generation, presumably by enhancing NOS activation by $\mathrm{Ca}^{2+}$.

Previous studies indicate that hippocampal neurons express the NOX2 enzyme (111), and that activation of NMDA receptors results in sequential activation of NOS and NOX2 in neocortical neuronal cultures (33). Here, we found that BDNF-induced cytoplasmic $\mathrm{H}_{2} \mathrm{O}_{2}$ generation required both NOS and NOX activities. Previous reports indicate that NOSgenerated nitric oxide enhances RyR activity via $S$-nitrosylation of RyR cysteine residues (109); however, ROS-induced RyR redox modifications, and not RyR $S$-nitrosylation, are required for brain RyR channel stimulation promoted by increased

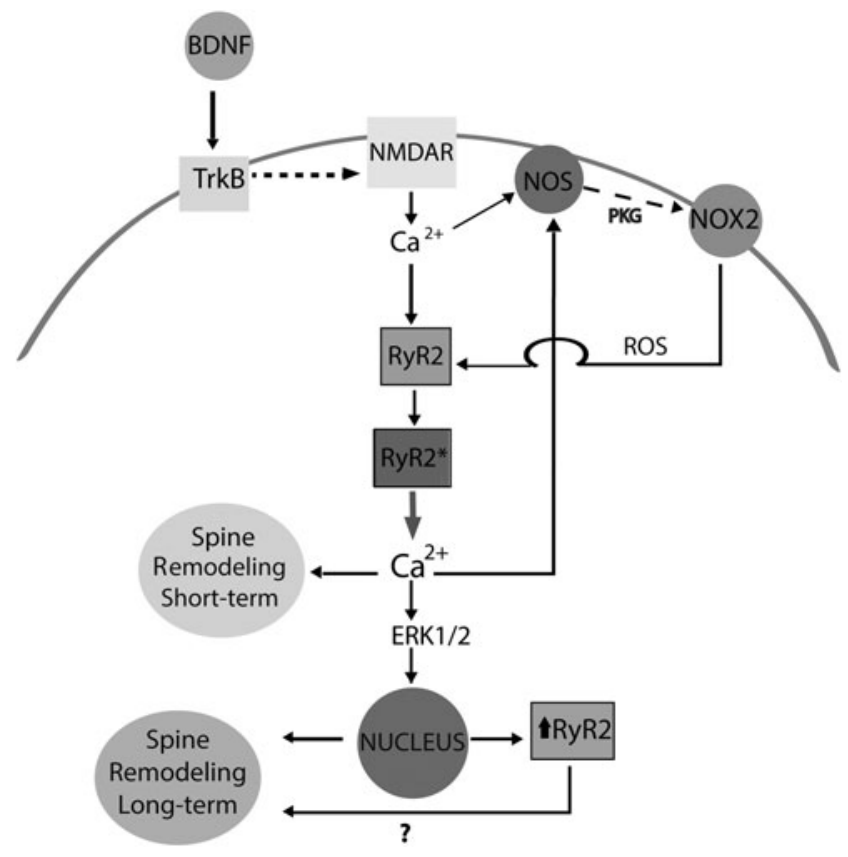

FIG. 10. Scheme of the model proposed to account for RyR2-dependent spine remodeling and $R y R 2$ upregulation. The figure illustrates a model through which BDNF binding to its membrane receptor TrkB triggers a signaling cascade that engages sequentially NMDA receptors and the NOS, PKG, and NOX2 enzymes, resulting in increased ROS generation. The increase in $\mathrm{Ca}^{2+}$ produced by NMDA receptor activation together with NOX2-generated ROS enhances RyR2 activity, indicated as RyR2* in the figure. The resulting enhanced $\mathrm{Ca}^{2+}$ signaling, generated via redoxdependent CICR, promotes short- and long-term spine remodeling, the latter via $\mathrm{Ca}^{2+}$-dependent ERK1/2 activation, and results in RyR2 upregulation. The question mark illustrated in the scheme reflects that it is not known whether RyR2 upregulation contributes to long-term spine remodeling. CICR, $\mathrm{Ca}^{2+}$-induced $\mathrm{Ca}^{2+}$ release. cellular oxidative conditions (20). Our results show that PKG inhibition suppressed BDNF-induced ROS generation. Hence, we propose that BDNF triggers a signaling cascade that engages sequentially NMDA receptors, NOS, PKG, and NOX2 enzymes, and redox-sensitive RyR2-mediated CICR, as illustrated in the scheme presented in Figure 10.

\section{BDNF-induced RyR2 upregulation}

Here, we show that RyR2 downregulation prevented $\mathrm{Ca}^{2+}$ release induced by 4-CMC, a strong indication that the RyR2 isoform is mostly responsible for this response. RyR2 downregulation hindered BDNF-induced RyR2 upregulation as well, suggesting that RyR2-mediated $\mathrm{Ca}^{2+}$ release is a key factor for its own expression. In addition, we found that the enhanced RyR 2 expression induced by BDNF required ERK1/2 activity, which has a key role in the synaptic plasticity processes elicited by BDNF (4). Moreover, ERK1/2 activity is essential for the activation of the transcription factor cAMP response elementbinding (CREB), which is central for long-term hippocampal synaptic plasticity and spatial memory processes (7). Nicotine administration to mice upregulates RyR2 levels in a number of brain areas associated with cognition and addiction, such as the cortex and ventral midbrain; RyR2 upregulation requires CREB activation via a mechanism dependent on CICR (122).

Future studies should address if BDNF-induced RyR2 upregulation involves CREB activation in hippocampal neurons. In addition, we found that the antioxidant NAC, or NOS or NOX2 inhibition, prevented BDNF-induced RyR2 upregulation. We suggest that cross talk between RyR2-mediated $\mathrm{Ca}^{2+}$ and ROS signaling plays a key role in RyR2 upregulation induced by BDNF.

\section{BDNF-induced dendritic spine remodeling}

Dendritic spines are miniature protrusions that compose most excitatory synapses in the mammalian brain [reviewed in Lippman and Dunaevsky (71) and Nimchinsky et al. (86)]. These structures receive, integrate, and compartmentalize the immense majority of excitatory inputs $(37,86)$. The shape and numbers of spines are a direct consequence of changes in neuronal activity $(15,112)$, which engages different $\mathrm{Ca}^{2+}$ signaling pathways $(39,42,103)$. Dendritic spine remodeling, including the formation/elongation and the pruning/retraction of spines, requires changes in intracellular $\mathrm{Ca}^{2+}$ concentration (87), which are necessary for the actin-mediated spine enlargement associated with electrical stimulation $(30,87,114)$. Previous work demonstrated that BDNF has an essential role in the structural remodeling of excitatory synapses $(3,4,113)$. In brain slices, the gradual spine enlargement induced specifically by synaptic stimulation strongly depends on protein synthesis and BDNF signaling (110). Dendritic spine size positively correlates with an increase in AMPA-receptormediated currents at stimulated synapses and depends on NMDA receptors, calmodulin and actin polymerization (77, 78). In young hippocampal synapses, increased or decreased spine sizes, respectively, occur during persistent LTP or longterm depression $(78,82,89,120)$. Spontaneously released glutamate is sufficient to activate nearby spines, which can then lead to the growth of new postsynaptic processes connecting to a presynaptic site (100). Similarly, in cultured hippocampal neurons, short pulses of glutamate applied to 
dendrites induce spine elongation, whereas long pulses cause shrinking (59).

One of the main findings of our work is the strong dependence of BDNF-induced dendritic spine remodeling on RyR2-mediated $\mathrm{Ca}^{2+}$ release and ROS generation via NOS/ NOX2. These results, which add to previous work $(1,58)$, show for the first time that BDNF-induced ROS generation is important for the structural remodeling of dendritic spines induced by BDNF. In addition, RyR2-mediated $\mathrm{Ca}^{2+}$ signals also mediated BDNF-induced dendritic spine remodeling, highlighting that the cross talk between $\mathrm{Ca}^{2+}$ and ROS signaling is a central process in activity-dependent neuronal function. In fact, ROS signaling and RyR-mediated $\mathrm{Ca}^{2+}$ release are key activities for neuronal polarization (115).

\section{RyR2 downregulation impairs spatial memory}

The involvement of RyR channels in the generation of postsynaptic $\mathrm{Ca}^{2+}$ signals, synaptic plasticity, and memory processes has acquired increasing relevance in the last two decades $(8,28,29,31,74,81,97,99,101,119)$. In chickens trained in a passive avoidance discrimination task, treatment with dantrolene to inhibit RyR channel activity causes loss of memory retention (28), while administration of the RyR agonist 4-CMC enhances memory consolidation (8). In mice conditioned in an inhibitory avoidance task (31), or trained in a radial arm-maze task (88), RyR channel inhibition with dantrolene or with high concentrations of ryanodine alters memory retention. In contrast, intrahippocampal injection of stimulatory ryanodine enhances both learning and memory consolidation in rats trained in the Morris water maze (1). Selective knockdown by intracerebrovascular injection of antisense ODNs of the RyR2 and the RyR3 isoforms in mice, but not of the RyR1 isoform, impairs memory retention in a passive avoidance test (31).

Here, we report that injection in the hippocampal CA1 region of a specific ODN anti-RyR2 caused severe defects in a spatial task previously memorized by rats. Remarkably, rats exhibited utter disorientation after downregulation of RyR2 expression, while injection of the scrambled ODN did not affect the memorized behavior. These results highlight the relevance of RyR2 expression for successful performance of hippocampal-dependent spatial tasks. Furthermore, rodent exposure to different hippocampal-dependent memory tasks, including the Morris water maze $(1,119)$ and novel object recognition (6), results in significant RyR2 upregulation and in less prominent RyR3 upregulation as well. Based on these combined findings, it seems reasonable to propose that hippocampal-dependent memory formation and consolidation not only require RyR2 channel expression but also entail RyR2 $>$ RyR3 upregulation to ensure the generation of the $\mathrm{Ca}^{2+}$ signals required for memory processes.

It is worth mentioning that sublethal concentrations of soluble amyloid $\beta$-peptide oligomers (A $\beta \mathrm{Os})$, increasingly recognized as causative agents of Alzheimer's disease, decrease RyR2 protein content and prevent BDNF- or caffeine-induced spine remodeling (92). The significant RyR2 protein downregulation induced by $\mathrm{A} \beta \mathrm{Os}$ may contribute to the RyR decrease reported in Alzheimer's patients at early stages of the disease (51). Hence, RyR2 downregulation may represent a significant factor in the impaired synaptic plasticity and memory defects characteristic of this neurodegenerative disorder.

\section{Materials and Methods}

\section{Materials}

BDNF and 4-CMC were from Merck Millipore (Darmstadt, Germany). Fluo4-AM, calcein-AM, Alexa Fluor ${ }^{\circledR} 488$, TRIzol reagent Hoechst 33258, GlutaMAX ${ }^{\mathrm{TM}}$, and Lipofectamine 2000 transfection reagent were from Invitrogen (Carlsbad, CA). DAKO mounting medium and Brilliant III Ultra-Fast SYBR $^{\circledR}$ GREEN qPCR Master Mix were from Agilent Technologies (Santa Clara, CA). Ryanodine was from Tocris Bioscience (Bristol, United Kingdom). N-acetyl-L-cysteine, L-NAME, and MK801 were from Sigma-Aldrich (Darmstadt, Germany). KT-5823 was from Tocris Bioscience. Gp91ds-tat and gpscr were from Anaspec (Fremont, CA). B27 supplement and Neurobasal medium were from Gibco (Carlsbad, CA). Mounting solution for slide coating was from Vectashield (Vector Laboratories, Burlingame, CA).

Mouse anti-RyR2 (MA3-916) was from Thermo Fisher. Rabbit anti-RyR3 (AB9082) was from Merck Millipore, and monoclonal anti- $\beta$ actin (A5316) from Sigma-Aldrich (St. Louis, MO). A highly selective RyR1 antibody was kindly provided by Dr. Vincenzo Sorrentino. DNAase digestion (DNA-free ${ }^{T M} \mathrm{Kit}$ ) was obtained from Ambion (Austin, TX), and ImProm-II Reverse Transcriptase kit was from Promega (Madison, WI). PVDF membranes (Trans-Blot ${ }^{\circledR}$ Turbo Mini PVDF) were from Bio-Rad Laboratories (Hercules, CA). The amplification system MX3000P was from Stratagene (La Jolla, CA). The pRFP-C-RS and pRFP-C-RS (TR30014) vectors and plasmid containing a noneffective 29mer shScr cassette (TR30013 and TR30015) were from Origene (Rockville, MA). The HyPer ${ }^{\mathrm{TM}}$-Cyto plasmid was from Evrogen (Moscow, Russia).

\section{Primary rat hippocampal cultures}

Primary cultures were prepared from the hippocampus dissected from Sprague-Dawley rats at embryonic day 18 (1, 92). Cells were plated in minimum essential medium plus $10 \%$ horse serum for $40 \mathrm{~min}$ to allow adhesion of neurons and minimize glial cell adhesion. Cultures were maintained subsequently at $37^{\circ} \mathrm{C}$ under $5 \% \mathrm{CO}_{2}$ in serum-free Neurobasal medium supplemented with B27 serum free and $2 \mathrm{mM}$ GlutaMAX. Hippocampal cultures were used at 14 days in vitro (DIV) for all experiments.

All experimental protocols used in this work complied with the "Guiding Principles for Research Involving Animals and Human Beings" of the American Physiological Society and were approved by the Bioethics Committee on Animal Research, Faculty of Medicine, Universidad de Chile.

\section{Transfection with knockdown plasmid vectors}

Cells in primary culture were transiently transfected with Lipofectamine 2000 according to the manufacturer's specifications. Neurons were transfected with $1 \mu \mathrm{L}$ Lipofectamine2000 in Neurobasal medium containing $2 \mu \mathrm{g}$ of shRNA expression vector specific to silence RyR2 expression. This shRNA expression vector was obtained by cloning a shRNA cassette directed against rat RyR2 mRNA (target sequence 5'-AGGAAGCAATGGTGGACAAGTTGGCTGAA-3') in the BamHI/HindIII cloning sites of pGFP-V-RS vector or pRFP-C-RS vector. A plasmid containing a noneffective 29mer shScr cassette (TR30013 and TR30015) was used as 
control. One day post-transfection, cultures were stimulated with BDNF $(50 \mathrm{ng} / \mathrm{mL})$ as described in the text to perform experiment of RyR2 expression or spine remodeling.

\section{RNA isolation and real time $P C R$}

Total RNA was isolated using TRIzol reagent; DNase digestion was included to remove any contaminating genomic DNA. RNA purity was assessed by the 260/280 absorbance ratio and RNA integrity by gel electrophoresis. Complementary DNA (cDNA) was synthesized with $1 \mu \mathrm{g}$ total RNA with the Improm TM II reverse transcriptase. One hundred nanograms of cDNA was used in $20 \mu \mathrm{L}$ final volume for qPCR amplification. qPCR was performed in an amplification system (MX3000P; Stratagene) using the Brilliant III Ultra-Fast SYBR GREEN qPCR Master Mix. Amplification was performed after $35 \mathrm{cy}-$ cles, each of which included $15 \mathrm{~s}$ at $95^{\circ} \mathrm{C}, 15 \mathrm{~s}$ at $60^{\circ} \mathrm{C}$ (RyR2 and $\beta$-actin), and $15 \mathrm{~s}$ at $72^{\circ} \mathrm{C}$. After these cycles, a final 15 -s incubation step at $95^{\circ} \mathrm{C}$ was added. Primers (1) were as follows (f: forward; r: reverse): RyR1 f 5'-GCCTTTGATGTGGGA TTACAG-3'; r 5'-CCCCAACTCGAACCTTCTCTC-3'. RyR2 f 5'-AATCAAAGTGGCGGAATTTCTTG-3'; r 5'-TCTCCCT CAGCCTTCTCCGGTTC-3'. RyR 3 f 5'-GAAGCCTGTTGGT GGACCATAC-3'; r 5'-TCCAGAGTGTTTGCATAAAGGA G-3'. $\beta$-actin f $5^{\prime}$-TCTACAATGAGCTGCGTGTG-3'; $\beta$-actin r 5'-TACATGGCTGGGGTGTTGAA-3'. The levels of RyR mRNA were assessed with the $2^{-\Delta \Delta \mathrm{CT}}$ method (95) using $\beta$ actin as housekeeping gene. Dissociation curves were analyzed to verify purity of products. All samples, including controls, were run in triplicate.

\section{Western blot analysis}

Cells extracts prepared as described (52) were resolved by sodium dodecyl sulfate/polyacrylamide gel electrophoresis ( $4 \%$ with a base of $15 \%$ polyacrylamide gels), transferred to PDVF membranes, and incubated overnight with specific antibodies against RyR2. To correct for loading, membranes were stripped and reprobed for $\beta$-actin using a specific antibody. The image acquisition and densitometric analysis of band density were performed by means of the ChemiDoc ${ }^{\mathrm{TM}}$ MP System and the Image Lab software by Bio-Rad Laboratories, respectively.

\section{Immunofluorescence}

Hippocampal cultures transfected with shRyR2 or shScr were fixed by adding an equal volume of $4 \%$ paraformaldehyde and Neurobasal medium for $5 \mathrm{~min}$. After replacing this medium with a solution containing $4 \%$ paraformaldehyde, cultures were incubated for $10 \mathrm{~min}$ and washed with phosphate-buffered saline (PBS) at $\mathrm{pH}$ 7.4. Fixed cultures were incubated for $2 \mathrm{~h}$ with a blocking and permeabilization solution containing 3\% donkey serum and $0.25 \%$ Triton X-100 (in PBS $1 \times$ ); next, cultures were incubated overnight at $4^{\circ} \mathrm{C}$ with primary antibody antiRyR2 diluted in blocking solution. After this incubation period, cultures were washed with PBS and incubated for $2 \mathrm{~h}$ with the secondary antibody Alexa Fluor 488 anti-rabbit (in blocking solution) at room temperature. Cultures were washed with PBS, and coverslips were mounted in DAKO mounting medium for microscope observation. Cells were visualized in a Nikon $\mathrm{C} 2+$ confocal microscope (Melville, NY), with the $63 \times$ objective lens. Images were analyzed using ImageJ software (National Institutes of Health).

\section{Immunohistochemistry}

Brain tissue fixation and immunohistochemistry were performed as described (1). Briefly, each animal was perfused transcardially and under anesthesia with saline solution $(\mathrm{NaCl} 0.9 \%)$, followed by $4 \%$ paraformaldehyde in $0.1 \mathrm{M}$ phosphate buffer, $\mathrm{pH} 7.4$, to fix and remove their brains. Brains were postfixed for $2 \mathrm{~h}$ in paraformaldehyde and were cryopreserved in PBS containing 30\% sucrose plus $0.03 \%$ sodium azide for subsequent slice preparation with a microtome. Coronal slices $(40 \mu \mathrm{m})$ were incubated in the blocking/ permeabilization solution for $2 \mathrm{~h}$ at room temperature, and were then incubated overnight at $4^{\circ} \mathrm{C}$ in blocking/permeabilization solution under shaking with anti-RyR2 or anti-RyR3 primary antibodies, diluted to $1: 50$ and 1:100, respectively. Slices were washed three times for $5 \mathrm{~min}$ with PBS, followed by a 5-min wash with blocking/permeabilization solution before further incubation with the secondary antibodies Alexa 488 goat anti-mouse and Alexa 488 chicken anti-rabbit, respectively. Slices were then washed three times with PBS, for $10 \mathrm{~min}$ at room temperature, and then incubated with Hoechst 33258 for detection of the cell nucleus for $5 \mathrm{~min}$ at room temperature. Slices were mounted on slides coated with mounting solution to preserve fluorescence. The ImageJ software program was used for image analysis and generation of zeta projections from stacks (1.5 mm thickness each). Total intensity of RyR2 or RyR3 fluorescence in each $z$ projection was measured and normalized by Hoechst fluorescence intensity.

\section{Determination of cytoplasmic $\mathrm{Ca}^{2+}$ signals}

Cells were transferred to modified Tyrode's solution (in $\mathrm{m} M: 129 \mathrm{NaCl}, 5 \mathrm{KCl}, 2 \mathrm{CaCl}_{2}, 1 \mathrm{MgCl}_{2}, 30$ glucose, and 25 HEPES, pH 7.3), preloaded for $30 \mathrm{~min}$ at $37^{\circ} \mathrm{C}$ with $2.5 \mu \mathrm{M}$ Fluo-4 AM, and washed for $10 \mathrm{~min}$ in modified Tyrode's solution to allow complete dye de-esterification. Fluorescence images of intracellular $\mathrm{Ca}^{2+}$ signals in primary hippocampal neurons (14 DIV) were obtained every $5 \mathrm{~s}$ with an inverted confocal microscope (Axiovert 200, LSM 5 Pascal; Carl Zeiss, Oberkochen, Germany), using Plan Apochromat $63 \times$ Oil DIC objective (excitation at $488 \mathrm{~nm}$, argon laser beam). Image data were acquired from different regions of optical interest located in the cell bodies. Frame scans were averaged using the equipment data acquisition program. Fluorescence signals are shown as $F / F_{0}$ values, where $F_{0}$ corresponds to the basal fluorescence recorded before any treatment. The increase in fluorescence induced by $0.5 \mathrm{mM} 4$ CMC did not saturate the probe, as tested by addition of ionomycin at the end of the experiment (2). All experiments were done at room temperature $\left(20-22^{\circ} \mathrm{C}\right)$.

\section{Determination of $\mathrm{H}_{2} \mathrm{O}_{2}$}

Hippocampal neurons were transiently transfected with the HyPer-Cyto plasmid at 14 DIV. This plasmid codes for a cytoplasmic protein, HyPer-Cyto, which has a circularly permuted yellow fluorescent protein inserted into the regulatory domain of the prokaryotic $\mathrm{H}_{2} \mathrm{O}_{2}$-sensing protein OxyR (12), allowing selective detection of $\mathrm{H}_{2} \mathrm{O}_{2}$ production in living cells. One day post-transfection, cells were washed with Tyrode's solution and were stimulated with BDNF $50 \mathrm{ng} / \mathrm{mL}$ in the same solution. Images were obtained every $5 \mathrm{~s}$ with an inverted 
confocal microscope (Axiovert 200, LSM 5 Pascal; Carl Zeiss) using the Plan Apochromat $63 \times$ Oil DIC objective (excitation at $488 \mathrm{~nm}$, argon laser beam). Changes in probe fluorescence, which reflect $\mathrm{H}_{2} \mathrm{O}_{2}$ levels, are presented as $F / F_{0}$ values, where $F_{0}$ corresponds to the average basal fluorescence obtained from a specific interest region in the soma.

\section{Morphological analysis of dendritic spines}

We analyzed the changes in spine morphology by confocal microscopy of hippocampal cultures (14 DIV). Confocal fluorescence images were obtained with a Zeiss LSM-5, Pascal 5 Axiovert 200 microscope, by using LSM 53.2 image capture and analysis software and a Plan-Apochromat $63 \times / 1.4$ Oil DIC objective. Dendrites in $30-50 \mu \mathrm{m}$ proximity to the soma were selected randomly independently of their spine density. We used the ImageJ software program for image deconvolution and zeta-projection reconstruction from 10 to 15 stacks $(0.4 \mu \mathrm{m}$ each $)$ to measure spine length. Spine density in dendrites was analyzed by measuring the number of spines present in a length of $50 \mu \mathrm{m}$; five dendrites were analyzed per condition in five independent experiments. Short-term changes were monitored for $1 \mathrm{~h}$ after BDNF incubation through acquisition of dendrite images every $15 \mathrm{~min}$; long-term changes were visualized after $6 \mathrm{~h}$ of incubation with BDNF. We used two different strategies to visualize cellular morphology; neurons were loaded for 20 min with $1 \mu M$ calcein-AM in Tyrode's solution or neurons were transfected with pGFP-V-RS or pRFP-C-RS vectors that express the green fluorescent protein (GFP) or the RFP, respectively, coupled to the expression of shRyR2. Transfection with the scrambled sequence or only with the RFP or GFP was used as control.

\section{Spatial memory evaluation}

Bilateral injections of ODN-RyR2 or ODN-Scr were performed into rat CA1 hippocampal region. Two-month-old Sprague-Dawley male rats (250-280 g) were anesthetized with isoflurane (3\% in oxygen) for induction of anesthesia. Isoflurane ( $2 \%$ in oxygen) was also used for maintenance of anesthesia, at a constant oxygen flow $(1 \mathrm{~L} / \mathrm{min})$. Two 21-gauge stainless steel guide cannulas were implanted bilaterally in the CA1 hippocampal region of each rat. Target coordinates, calculated as given in Ref. (94), were as follows relative to bregma: anteroposterior -3.30 , lateral $\pm 2.6 \mathrm{~mm}$, $1.8 \mathrm{~mm}$ in depth, with $10^{\circ}$ angles to target the dorsal CA1 hippocampal region. Guide cannulas were fixed to the skull with skull screws and dental cement. Following surgery, animals were individually housed with food and water ad libitum for 7 days before exposure to the Oasis maze task, a modified dryland version of the Morris water maze $(25,53)$.

To evaluate hippocampal-dependent spatial learning, cannulated and water-deprived pretrained animals were trained and tested during three consecutive daily sessions in the Oasis maze task, which involved searching for the reward (water) placed in the same one of 21 equidistant wells on a circular arena provided with visual cues; each session was composed of 15 trials of 1min duration each. After this initial training period, animals received three bilateral injections $(1.0 \mu \mathrm{L}$ each) of ODN-RyR2 $(10 \mathrm{nmol} / \mu \mathrm{L})$ or ODN-Scr $(10 \mathrm{nmol} / \mu \mathrm{L})$, with the following sequences. ODN-RyR2: 5'T*T*CGCCCGCATCAGCC*A*T-
3'; ODN-Scr: $5^{\prime} \mathrm{C}^{*} \mathrm{G} * \mathrm{GCAGGAGTCTGTGC} * \mathrm{G}^{*} \mathrm{C}-3^{\prime}$, the $*$ indicates the phosphorothioate residues.

A complete scheme of training sessions and injections is illustrated in the Table presented in Figure 9B. Animal behavior was recorded with a video camera in the zenithal position. Video recordings were analyzed using the Virtual Dub software. The position of the animal was tracked and navigation was reconstructed and analyzed with a MATLAB (MathWorks) routine. Six hours after the conclusion of session 9, animals were perfused and their brains were removed and sliced in a frozen microtome for immunohistochemistry analysis of RyR2 and RyR3 (for details, see the Immunohistochemistry section). Fluorescence images, captured in an inverted confocal microscope $\mathrm{C} 2+$ Spectral Nikon Eclipse TI, were analyzed with the ImageJ software.

\section{Statistics}

Results are expressed as mean $\pm \mathrm{SE}$ from at least three independent experiments. Statistical significance was evaluated with the GraphPad Prism 7.0 Software (San Diego, CA). The particular analysis performed in each case, Student's $t$-test, one-way or two-way ANOVA, is detailed in the Results section and figure legends.

\section{Acknowledgments}

We thank Dr. Vincenzo Sorrentino, University of Siena, Italy, for his kind gift of a highly specific RyR1 antibody. This work was supported by FONDECYT (3120093, 11140580, 1140545,1150736 , and 1170053) and by BNI (P-09-015).

\section{Author Disclosure Statement}

No competing financial interests exist.

\section{References}

1. Adasme T, Haeger P, Paula-Lima AC, Espinoza I, CasasAlarcón MM, Carrasco MA, and Hidalgo C. Involvement of ryanodine receptors in neurotrophin-induced hippocampal synaptic plasticity and spatial memory formation. Proc Natl Acad Sci U S A 108: 3029-3034, 2011.

2. Adasme T, Paula-lima A, and Hidalgo C. Inhibitory ryanodine prevents ryanodine receptor-mediated $\mathrm{Ca}^{2+}$ release without affecting endoplasmic reticulum $\mathrm{Ca}^{2+}$ content in primary hippocampal neurons. Biochem Biophys Res Commun 458: 57-62, 2015.

3. Alonso M, Vianna MR, Depino AM, Mello e Souza T, Pereira P, Szapiro G, Viola H, Pitossi F, Izquierdo I, and Medina JH. BDNF-triggered events in the rat hippocampus are required for both short- and long-term memory formation. Hippocampus 12: 551-560, 2002.

4. Alonso M, Medina JH, and Pozzo-miller L. ERK1/2 activation is necessary for BDNF to increase dendritic spine density in hippocampal CA1 pyramidal neurons. Learn Mem 11: 172-178, 2004.

5. Amaral MD and Pozzo-Miller L. BDNF induces calcium elevations associated with IBDNF, a nonselective cationic current mediated by TRPC channels. J Neurophysiol 98: 2476-2482, 2007.

6. Arias-Cavieres A, Adasme T, Sánchez G, Muñoz P, and Hidalgo C. Aging impairs hippocampal-dependent recognition memory and LTP and prevents the associated RyR up-regulation. Front Aging Neurosci 9: 1-16, 2017. 
7. Bading $\mathrm{H}$. Nuclear calcium signalling in the regulation of brain function. Nat Rev Neurosci 14: 593-608, 2013.

8. Baker KD, Edwards TM, and Rickard NS. A ryanodine receptor agonist promotes the consolidation of long-term memory in young chicks. Behav Brain Res 206: 143-146, 2010.

9. Baker KD, Edwards TM, and Rickard NS. The role of intracellular calcium stores in synaptic plasticity and memory consolidation. Neurosci Biobehav Rev 37: 12111239, 2013.

10. Balkowiec A and Katz DM. Cellular mechanisms regulating activity-dependent release of native brain-derived neurotrophic factor from hippocampal neurons. $\mathrm{J} \mathrm{Neu-}$ rosci 22: 10399-10407, 2002.

11. Bekinschtein P, Cammarota M, Igaz LM, Bevilaqua LR, Izquierdo I, and Medina JH. Persistence of long-term memory storage requires a late protein synthesis- and BDNF-dependent phase in the hippocampus. Neuron 53: 261-277, 2007.

12. Belousov VV, Fradkov AF, Lukyanov KA, Staroverov DB, Shakhbazov KS, Terskikh AV, and Lukyanov S. Genetically encoded fluorescent indicator for intracellular hydrogen peroxide. Nat Methods 3: 281-286, 2006.

13. Berridge MJ. Neuronal calcium signaling. Neuron 21: 1326, 1998.

14. Bibel M and Barde YA. Neurotrophins: key regulators of cell fate and cell shape in the vertebrate nervous system. Genes Dev 14: 2919-2937, 2000.

15. Bloodgood BL, Giessel AJ, and Sabatini BL. Biphasic synaptic $\mathrm{Ca}$ influx arising from compartmentalized electrical signals in dendritic spines. PLoS Biol 7: e1000190, 2009.

16. Blum $\mathrm{R}$ and Konnerth A. Neurotrophin-mediated rapid signaling in the central nervous system: mechanisms and functions. Physiology (Bethesda) 20: 70-78, 2005.

17. Blum R, Kafitz KW, and Konnerth A. Neurotrophinevoked depolarization requires the sodium channel NaV1.9. Nature 419: 687-693, 2002.

18. Bolton MM, Pittman AJ, and Lo DC. Brain-derived neurotrophic factor differentially regulates excitatory and inhibitory synaptic transmission in hippocampal cultures. J Neurosci 20: 3221-3232, 2000.

19. Bramham CR and Messaoudi E. BDNF function in adult synaptic plasticity: the synaptic consolidation hypothesis. Prog Neurobiol 76: 99-125, 2005.

20. Bull R, Finkelstein JP, Gálvez J, Sánchez G, Donoso P, Behrens MI, and Hidalgo C. Ischemia enhances activation by $\mathrm{Ca}^{2+}$ and redox modification of ryanodine receptor channels from rat brain cortex. J Neurosci 28: 9463-9472, 2008.

21. Burette A, Zabel U, Weinberg RJ, Schmidt HH, and Valtschanoff JG. Synaptic localization of nitric oxide synthase and soluble guanylyl cyclase in the hippocampus. J Neurosci 22: 8961-8970, 2002.

22. Carmignoto G, Pizzorusso T, Tia S, and Vicini S. Brainderived neurotrophic factor and nerve growth factor potentiate excitatory synaptic transmission in the rat visual cortex. J Physiol 498: 153-164, 1997.

23. Caroni P, Donato F, and Muller D. Structural plasticity upon learning: regulation and functions. Nat Rev Neurosci 13: 478-490, 2012.

24. Citri A and Malenka RC. Synaptic plasticity: multiple forms, functions, and mechanisms. Neuropsychopharmacology 33: 18-41, 2008.
25. Clark RE, Broadbent NJ, and Squire LR. Hippocampus and remote spatial memory in rats. Hippocampus 15: 260272, 2005.

26. Crozier RA, Black IB, and Plummer MR. Blockade of NR2B-containing NMDA receptors prevents BDNF enhancement of glutamatergic transmission in hippocampal neurons. Learn Mem 6: 257-266, 1999.

27. Edelmann E, Cepeda-Prado E, Franck M, Lichtenecker P, Brigadski $\mathrm{T}$, and Leßmann V. Theta burst firing recruits BDNF release and signaling in postsynaptic CA1 neurons in spike-timing-dependent LTP. Neuron 86: 1041-1054, 2015.

28. Edwards TM and Rickard NS. Pharmaco-behavioural evidence indicating a complex role for ryanodine receptor calcium release channels in memory processing for a passive avoidance task. Neurobiol Learn Mem 86: 1-8, 2006.

29. Emptage N, Bliss TV, and Fine A. Single synaptic events evoke NMDA receptor-mediated release of calcium from internal stores in hippocampal dendritic spines. Neuron 22: 115-124, 1999.

30. Fifková E. A possible mechanism of morphometric changes in dendritic spines induced by stimulation. Cell Mol Neurobiol 5: 47-63, 1985.

31. Galeotti N, Quattrone A, Vivoli E, Norcini M, Bartolini A, and Ghelardini C. Different involvement of type 1, 2, and 3 ryanodine receptors in memory processes. Learn Mem 15: 315-323, 2008.

32. Giannini G, Conti A, Mammarella S, and Scrobogna M. The ryanodine receptor/calcium channel genes are widely and differentially expressed in murine brain and peripheral tissues. J Cell Biol 128: 893-904, 1995.

33. Girouard H, Wang G, Gallo EF, Anrather J, Zhou P, Pickel VM, and Iadecola C. NMDA receptor activation increases free radical production through nitric oxide and NOX2. J Neurosci 29: 2545-2552, 2009.

34. Gordeeva AV, Zvyagilskaya RA, and Labas YA. Cross talk between reactive oxygen species and calcium in living cells. Biochemistry (Mosc) 68: 1077-1080, 2003.

35. Gôrlach A, Bertram K, Hudecova S, and Krizanova O. Calcium and ROS: a mutual interplay. Redox Biol 6: 260271, 2015.

36. Gottmann K, Mittmann T, and Lessmann V. BDNF signaling in the formation, maturation and plasticity of glutamatergic and GABAergic synapses. Exp Brain Res 199: 203-234, 2009.

37. Harris K and Kater SB. Dendritic spines: cellular specializations imparting both stability and flexibility to synaptic function. Annu Rev Neurosci 17: 341-371, 1994.

38. Hartmann M, Heumann R, and Lessmann V. Synaptic secretion of BDNF after high-frequency stimulation of glutamatergic synapses. EMBO J 20: 5887-5897, 2001.

39. Hayashi Y and Majewska AK. Dendritic spine geometry: functional implication and regulation. Neuron 46: 529532, 2005.

40. He J, Gong H, and Luo Q. BDNF acutely modulates synaptic transmission and calcium signalling in developing cortical neurons. Cell Physiol Biochem 16: 69-76, 2005.

41. Hidalgo $\mathrm{C}$ and Donoso P. Crosstalk between calcium and redox signaling: from molecular mechanisms to health implications. Antioxid Redox Signal 10: 1275-1312, 2008.

42. Holcman D, Schuss Z, and Korkotian E. Calcium dynamics in dendritic spines and spine motility. Biophys $J$ 87: 81-91, 2004. 
43. Holtmaat A and Svoboda K. Experience-dependent structural synaptic plasticity in the mammalian brain. Nat Rev Neurosci 10: 759, 2009.

44. Huang EJ and Reichardt LF. Trk receptors: roles in neuronal signal transduction. Annu Rev Biochem 72: 609-642, 2003.

45. Itami C, Kimura F, Kohno T, Matsuoka M, Ichikawa M, Tsumoto T, and Nakamura S. Brain-derived neurotrophic factor-dependent unmasking of "silent" synapses in the developing mouse barrel cortex. Proc Natl Acad Sci U S A 100: 13069-13074, 2003.

46. Johenning FW, Theis AK, Pannasch U, Rückl M, Rüdiger $\mathrm{S}$, and Schmitz D. Ryanodine receptor activation induces long-term plasticity of spine calcium dynamics. PLOS Biol 13: e1002181, 2015.

47. Kafitz KW, Rose CR, Thoenen H, and Konnerth A. Neurotrophin-evoked rapid excitation through TrkB receptors. Nature 401: 918-921, 1999.

48. Kamsler A and Segal M. Hydrogen peroxide as a diffusible signal molecule in synaptic plasticity. Mol Neurobiol 29: 167-178, 2004.

49. Kamsler A and Segal M. Paradoxical actions of hydrogen peroxide on long-term potentiation in transgenic superoxide dismutase-1 mice. J Neurosci 23: 10359-10367, 2003.

50. Kang $\mathrm{H}$ and Schuman EM. A requirement for local protein synthesis in neurotrophin-induced hippocampal synaptic plasticity. Science 273: 1402-1406, 1996.

51. Kelliher M, Fastbom J, Cowburn RF, Bonkale W, Ohm TG, Ravid R, Sorrentino V, and O'Neill C. Alterations in the ryanodine receptor calcium release channel correlate with Alzheimer's disease neurofibrillary and $\beta$-amyloid pathologies. Neuroscience 92: 499-513, 1999.

52. Kemmerling U, Muñoz P, Müller M, Sánchez G, Aylwin ML, Klann E, Carrasco MA, and Hidalgo C. Calcium release by ryanodine receptors mediates hydrogen peroxideinduced activation of ERK and CREB phosphorylation in N2a cells and hippocampal neurons. Cell Calcium 41: 491-502, 2007.

53. Kesner RP, Farnsworth G, and Kametani H. Role of parietal cortex and hippocampus in representing spatial information. Cereb Cortex 1: 367-373, 1991.

54. Kim SH, Won SJ, Sohn S, Kwon HJ, Lee JY, Park JH, and Gwag BJ. Brain-derived neurotrophic factor can act as a pronecrotic factor through transcriptional and translational activation of NADPH oxidase. J Cell Biol 159: 821-831, 2002.

55. Klöcker N, Cellerino A, and Bähr M. Free radical scavenging and inhibition of nitric oxide synthase potentiates the neurotrophic effects of brain-derived neurotrophic factor on axotomized retinal ganglion cells in vivo. $J$ Neurosci 18: 1038-1046, 1998.

56. Koh JY, Gwag BJ, Lobner D, and Choi DW. Potentiated necrosis of cultured cortical neurons by neurotrophins. Science 268: 573-575, 1995.

57. Kolarow R, Kuhlmann CRW, Munsch T, Zehendner C, Brigadski T, Luhmann HJ, and Lessmann V. BDNF-induced nitric oxide signals in cultured rat hippocampal neurons: time course, mechanism of generation, and effect on neurotrophin secretion. Front Cell Neurosci 8: 323, 2014.

58. Korkotian E. Fast confocal imaging of calcium released from stores in dendritic spines. Eur J Neurosci 10: 20762084, 1998.

59. Korkotian E and Segal M. Release of calcium from stores alters the morphology of dendritic spines in cultured hippocampal neurons. Proc Natl Acad Sci U S A 96: 12068-12072, 1999.

60. Korte M, Carroll P, Wolf E, Brem G, Thoenen H, and Bonhoeffer T. Hippocampal long-term potentiation is impaired in mice lacking brain-derived neurotrophic factor. Proc Natl Acad Sci U S A 92: 8856-8860, 1995.

61. Kovalchuk Y, Hanse E, Kafitz KW, and Konnerth A. Postsynaptic induction of BDNF-mediated long-term potentiation. Science 295: 1729-1734, 2002.

62. Kuczewski N, Porcher C, Ferrand N, Fiorentino H, Pellegrino C, Kolarow R, Lessmann V, Medina I, and Gaiarsa JL. Backpropagating action potentials trigger dendritic release of BDNF during spontaneous network activity. $J$ Neurosci 28: 7013-7023, 2008.

63. Leite MF, Burgstahler AD, and Nathanson $\mathrm{MH}$. $\mathrm{Ca}^{2+}$ waves require sequential activation of inositol trisphosphate receptors and ryanodine receptors in pancreatic acini. Gastroenterology 122: 415-427, 2002.

64. Lessmann V, Gottmann K, and Heumann R. BDNF and NT-4/5 enhance glutamatergic synaptic transmission in cultured hippocampal neurones. Neuroreport 6: 21-25, 1994.

65. Leßmann V and Heumann R. Modulation of unitary glutamatergic synapses by neurotrophin- $4 / 5$ or brain-derived neurotrophic factor in hippocampal microcultures: presynaptic enhancement depends on pre-established paired-pulse facilitation. Neuroscience 86: 399-413, 1998.

66. Levine ES, Crozier RA, Black IB, and Plummer MR. Brain-derived neurotrophic factor modulates hippocampal synaptic transmission by increasing N-methyl-D-aspartic acid receptor activity. Proc Natl Acad Sci U S A 95: 10235-10239, 1998.

67. Levine ES, Dreyfus CF, Black IB, and Plummer MR. Brain-derived neurotrophic factor rapidly enhances synaptic transmission in hippocampal neurons via postsynaptic tyrosine kinase receptors. Proc Natl Acad Sci U S A 92: 8074-8077, 1995.

68. Levine ES and Kolb JE. Brain-derived neurotrophic factor increases activity of NR2B-containing N-methyl-Daspartate receptors in excised patches from hippocampal neurons. J Neurosci Res 62: 357-362, 2000.

69. Li Y, Calfa G, Inoue T, Amaral MD, and Pozzo-Miller L. Activity-dependent release of endogenous BDNF from mossy fibers evokes a TRPC 3 current and $\mathrm{Ca}^{2+}$ elevations in CA3 pyramidal neurons. J Neurophysiol 103: 2846-2856, 2010.

70. Lin SY, Wu K, Levine ES, Mount HT, Suen PC, and Black IB. BDNF acutely increases tyrosine phosphorylation of the NMDA receptor subunit $2 \mathrm{~B}$ in cortical and hippocampal postsynaptic densities. Brain Res Mol Brain Res 55: 20-27, 1998.

71. Lippman J and Dunaevsky A. Dendritic spine morphogenesis and plasticity. J Neurobiol 64: 47-57, 2005.

72. Liu X, Ramirez S, Pang PT, Puryear CB, Govindarajan A, Deisseroth K, and Tonegawa S. Optogenetic stimulation of a hippocampal engram activates fear memory recall. Nature 484: 381-385, 2012.

73. Lohof AM, Ip NY, and Poo MM. Potentiation of developing neuromuscular synapses by the neurotrophins NT-3 and BDNF. Nat Lett 363: 350-353, 1993.

74. Lu YF and Hawkins RD. Ryanodine receptors contribute to cGMP-induced late-phase LTP and CREB phosphorylation in the hippocampus. J Neurophysiol 88: 1270-1278, 2002.

75. Mariga A, Zavadil J, Ginsberg SD, and Chao MV. Withdrawal of BDNF from hippocampal cultures leads to 
changes in genes involved in synaptic function. Dev Neurobiol 75: 173-192, 2015.

76. Massaad CA and Klann E. Reactive oxygen species in the regulation of synaptic plasticity and memory. Antioxid Redox Signal 14: 2013-2054, 2011.

77. Matsuzaki M, Ellis-Davies GCR, Nemoto T, Miyashita Y, Iino $\mathrm{M}$, and Kasai H. Dendritic spine geometry is critical for AMPA receptor expression in hippocampal CA1 pyramidal neurons. Nat Neurosci 4: 1086-1092, 2001.

78. Matsuzaki M, Honkura N, Ellis-Davies GCR, and Kasai H. Structural basis of long-term potentiation in single dendritic spines. Nature 429: 761-766, 2004.

79. Mizuno M, Yamada K, He J, Nakajima A, and Nabeshima T. Involvement of BDNF receptor TrkB in spatial memory formation. Learn Mem 10: 108-115, 2003.

80. Mizuno M, Yamada K, Olariu A, Nawa H, and Nabeshima $\mathrm{T}$. Involvement of brain-derived neurotrophic factor in spatial memory formation and maintenance in a radial arm maze test in rats. $J$ Neurosci 20: 7116-7121, 2000.

81. Muñoz P, Humeres A, Elgueta C, Kirkwood A, Hidalgo $C$, and Núñez MT. Iron mediates N-Methyl-D-aspartate receptor-dependent stimulation of calcium-induced pathways and hippocampal synaptic plasticity. J Biol Chem 286: 13382-13392, 2011.

82. Nägerl UV, Eberhorn N, Cambridge SB, and Bonhoeffer T. Bidirectional activity-dependent morphological plasticity in hippocampal neurons. Neuron 44: 759-767, 2004.

83. Nakazawa T, Komai S, Tezuka T, Hisatsune C, Umemori H, Semba K, Mishina M, Manabe T, and Yamamoto T. Characterization of Fyn-mediated tyrosine phosphorylation sites on GluR $\varepsilon 2$ (NR2B) subunit of the N-methyl-Daspartate receptor. J Biol Chem 276: 693-699, 2001.

84. Nguyen PV and Kandel ER. A macromolecular synthesisdependent late phase of long-term potentiation requiring cAMP in the medial perforant pathway of rat hippocampal slices. J Neurosci 16: 3189-3198, 1996.

85. Nguyen PV and Kandel ER. Brief theta-burst stimulation induces a transcription-dependent late phase of LTP requiring cAMP in area CA1 of the mouse hippocampus. Learn Mem 4: 230-243, 1997.

86. Nimchinsky EA, Sabatini BL, and Svoboda K. Structure and function of dendritic spines. Annu Rev Physiol 64: 313-353, 2002.

87. Oertner TG and Matus A. Calcium regulation of actin dynamics in dendritic spines. Cell Calcium 37: 477-482, 2005.

88. Ohnuki T and Nomura Y. 1-(((5-(4-Nitrophenyl)2furanyl)methylene)imino)-2,4-imidazolidinedione (dantrolene), an inhibitor of intracellular $\mathrm{Ca}^{2+}$ mobilization, impairs avoidance performance and spatial memory in mice. Biol Pharm Bull 19: 1038-1040, 1996.

89. Okamoto K, Nagai T, Miyawaki A, and Hayashi Y. Rapid and persistent modulation of actin dynamics regulates postsynaptic reorganization underlying bidirectional plasticity. Nat Neurosci 7: 1104-1112, 2004.

90. Oliveira AM and Bading H. Calcium signaling in cognition and aging-dependent cognitive decline. Biofactors 37: 168-174, 2011.

91. Park H and Poo MM. Neurotrophin regulation of neural circuit development and function. Nat Rev Neurosci 14: 7-23, 2013.

92. Paula-Lima AC, Adasme T, SanMartín C, Sebollela A, Hetz C, Carrasco MA, Ferreira ST, and Hidalgo C. Amyloid $\beta$-peptide oligomers stimulate RyR-mediated $\mathrm{Ca}^{2+}$ release inducing mitochondrial fragmentation in hippocampal neurons and prevent RyR-mediated dendritic spine remodeling produced by BDNF. Antioxid Redox Signal 14: 1209-1223, 2011.

93. Paula-Lima AC, Adasme T, and Hidalgo C. Contribution of $\mathrm{Ca}^{2+}$ release channels to hippocampal synaptic plasticity and spatial memory: potential redox modulation. Antioxid Redox Signal 21: 892-914, 2014.

94. Paxinos G and Watson C. The Rat Brain in Stereotaxic Coordinates. San Diego, CA: Academic Press, 1998, p. 34.

95. Pfaffl MW. A new mathematical model for relative quantification in real-time RT-PCR. Nucleic Acids Res 29: e45, 2001.

96. Pozzo-Miller LD, Gottschalk W, Zhang L, McDermott K, Du J, Gopalakrishnan R, Oho C, Sheng ZH, and Lu B. Impairments in high-frequency transmission, synaptic vesicle docking, and synaptic protein distribution in the hippocampus of BDNF knockout mice. J Neurosci 19: 4972-4983, 1999.

97. Raymond CR, and Redman SJ. Different calcium sources are narrowly tuned to the induction of different forms of LTP. J Neurophysiol 88: 249-255, 2002.

98. Rey FE, Cifuentes ME, Kiarash A, Quinn MT, and Pagano PJ. Novel competitive inhibitor of NAD(P)H oxidase assembly attenuates vascular $\mathrm{O}(2)(-)$ and systolic blood pressure in mice. Circ Res 89: 408-414, 2001.

99. Reyes-Harde M, Potter BV, Galione A, and Stanton PK. Induction of hippocampal LTD requires nitric-oxidestimulated PKG activity and $\mathrm{Ca}^{2+}$ release from cyclic ADP-ribose-sensitive stores. J Neurophysiol 82: 15691576, 1999.

100. Richards DA, Mateos JM, Hugel S, de Paola V, Caroni P, Gähwiler BH, McKinney RA. Glutamate induces the rapid formation of spine head protrusions in hippocampal slice cultures. Proc Natl Acad Sci U S A 102: 6166-6171, 2005.

101. Riquelme D, Alvarez A, Leal N, Adasme T, Espinoza I, Valdés JA, Troncoso N, Hartel S, Hidalgo J, Hidalgo C, and Carrasco MA. High-frequency field stimulation of primary neurons enhances ryanodine-receptor-mediated $\mathrm{Ca}^{2+}$ release and generates hydrogen peroxide, which jontly stimulate NF-kB activity. Antioxid Redox Signal 14: 1245-1259, 2011.

102. Rostas JA, Brent VA, Voss K, Errington ML, Bliss TV, and Gurd JW. Enhanced tyrosine phosphorylation of the 2B subunit of the N-methyl-D-aspartate receptor in longterm potentiation. Proc Natl Acad Sci U S A 93: 1045210456, 1996.

103. Sabatini BL, Oertner TG, and Svoboda K. The life cycle of $\mathrm{Ca}^{2+}$ ions in dendritic spines. Neuron 33: 439-452, 2002.

104. Samdani AF, Newcamp C, Resink A, Facchinetti F, Hoffman BE, Dawson VL, and Dawson TM. Differential susceptibility to neurotoxicity mediated by neurotrophins and neuronal nitric oxide synthase. J Neurosci 17: 46334641, 1997.

105. Segal RA and Greenberg ME. Intracellular signaling pathways activated by neurotrophic factors. Annu Rev Neurosci 19: 463-489, 1996.

106. Serrano F and Klann E. Reactive oxygen species and synaptic plasticity in the aging hippocampus. Ageing Res Rev 3: 431-443, 2004.

107. Sherwood NT and Lo DC. Long-term enhancement of central synaptic transmission by chronic brain-derived neurotrophic factor treatment. J Neurosci 19: 7025-7036, 1999. 
108. Suen PC, Wu K, Levine ES, Mount HT, Xu JL, Lin SY, and Black IB. Brain-derived neurotrophic factor rapidly enhances phosphorylation of the postsynaptic N-methylD-aspartate receptor subunit 1. Proc Natl Acad Sci US A 94: 8191-8195, 1997.

109. Sun J, Xin C, Eu JP, Stamler JS, and Meissner G. Cysteine-3635 is responsible for skeletal muscle ryanodine receptor modulation by NO. Proc Natl Acad Sci U S A 98: 11158-11162, 2001.

110. Tanaka J, Horiike Y, Matsuzaki M, Miyazaki T, EllisDavies GC, and Kasai H. Protein synthesis and neurotrophin-dependent structural plasticity of single dendritic spines. Science 319: 1683-1687, 2008.

111. Tejada-Simon MV, Serrano F, Villasana LE, Kanterewicz BI, Wu GY, Quinn MT, Klann E. Synaptic localization of a functional NADPH oxidase in the mouse hippocampus. Mol Cell Neurosci 29: 97-106, 2005.

112. Tsay D and Yuste R. On the electrical function of dendritic spines. Trends Neurosci 27: 77-83, 2004.

113. Tyler WJ, Alonso M, Bramham CR, and Pozzo-Miller LD. From acquisition to consolidation: on the role of brain-derived neurotrophic factor signaling in hippocampaldependent learning. Learn Mem 9: 224-237, 2002.

114. Vlachos A, Korkotian E, Schonfeld E, Copanaki E, Deller $\mathrm{T}$, and Segal M. Synaptopodin regulates plasticity of dendritic spines in hippocampal neurons. J Neurosci 29: 1017-1033, 2009.

115. Wilson C, Muñoz-Palma E, Henríquez DR, Palmisano I, Núñez MT, Di Giovanni S, and González-Billault C. A feed-forward mechanism involving the NOX complex and RyR-mediated $\mathrm{Ca}^{2+}$ release during axonal specification. $J$ Neurosci 36: 11107-11119, 2016.

116. Xu Z, Ji X, and Boysen PG. Exogenous nitric oxide generates ROS and induces cardioprotection: involvement of PKG, mitochondrial KATP channels, and ERK. Am J Physiol Heart Circ Physiol 286: HI433-HI440, 2004.

117. Yan Y, Chao-Liang W, Wan-rui Z, He-ping C, and Jie L. Cross-talk between calcium and reactive oxygen species signaling. Acta Pharmacol Sin 27: 821-826, 2006.

118. Zagrebelsky $\mathrm{M}$ and Korte M. Form follows function: BDNF and its involvement in sculpting the function and structure of synapses. Neuropharmacology 76: 628-638, 2014.

119. Zhao W, Meiri N, Xu H, Cavallaro S, Quattrone A, Zhang $\mathrm{L}$, and Alkon DL. Spatial learning induced changes in expression of the ryanodine type II receptor in the rat hippocampus. FASEB J 14: 290-300, 2000.

120. Zhou Q, Homma KJ, and Poo MM. Shrinkage of dendritic spines associated with long-term depression of hippocampal synapses. Neuron 44: 749-757, 2004.

121. Zima AV and Blatter LA. Redox regulation of cardiac calcium channels and transporters. Cardiovasc Res 71: 310-321, 2006.

122. Ziviani E, Lippi G, Bano D, Munarriz E, Guiducci S, Zoli M, Young KW, and Nicotera P. Ryanodine receptor-2 upregulation and nicotine-mediated plasticity. EMBO $J$ 30: 194-204, 2011.
Address correspondence to: Dr. Tatiana Adasme

Centro Integrativo de Biología y Química Aplicada Universidad Bernardo O'Higgins

General Gana 1702

Santiago 8370854

Chile

E-mail: tatiana.adasme@ubo.cl

Date of first submission to ARS Central, July 11, 2017; date of final revised submission, January 17, 2018; date of acceptance, January 20, 2018.

\begin{tabular}{|c|}
\hline 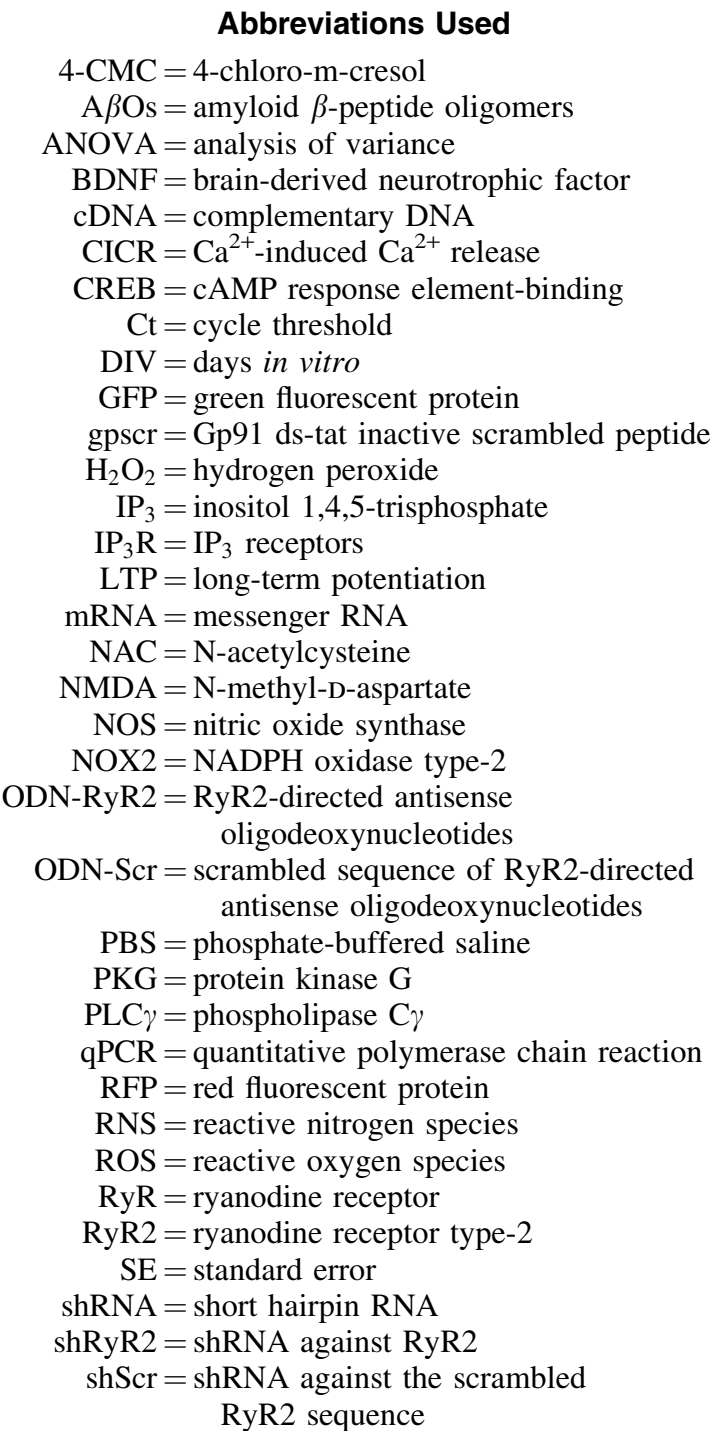 \\
\hline
\end{tabular}

